\title{
Modular Synthesis of Fluorous Trialkylphosphines
}

Gábor Vlád, ${ }^{\mathrm{a}}$ Frank Richter ${ }^{\mathrm{b}}$ and István T. Horváth*a

${ }^{a}$ Eötvös University, Department of Chemical Technology and Environmental Chemistry, Pázmány Péter sétány 1/A, H-1117 Budapest, Hungary.

${ }^{b}$ Bayer Material Science AG, Leverkusen, D-51368, Germany.

Supporting Information 


\section{Table of Contents}

3 Detailed Synthetic Protocol

$4 \quad$ NMR Data of Prepared Compounds

$10 \quad{ }^{31} \mathrm{P}\left\{{ }^{1} \mathrm{H}\right\}$-NMR Spectra of Prepared Compounds 


\section{Detailed Synthetic Protocol}

Typical procedure for the preparation of $\left[\mathrm{R}_{\mathrm{F} 8}\left(\mathrm{CH}_{2}\right)_{3}\right]_{3} \mathrm{P}: \quad \operatorname{Tris}(2-$ cyanoethyl)phosphine (5.257 g, $27.21 \mathrm{mmol})$ and $\mathrm{R}_{\mathrm{F} 8}\left(\mathrm{CH}_{2}\right)_{3} \mathrm{I}(27.00 \mathrm{~g}, 45.91 \mathrm{mmol})$ was placed into a round-bottomed flask, closed with a stop-cock, clamped, heated with vigorous stirring at $130^{\circ} \mathrm{C}$ for 1 hour, and then at $160^{\circ} \mathrm{C} 10$ hours. After cooling to $35^{\circ} \mathrm{C}$, $50 \mathrm{ml}$ of $\mathrm{CH}_{2} \mathrm{Cl}_{2}$ was added to the yellowish-brown solid, the mixture finely powdered in a mortar, and filtered through a Büchner-funnel. Washing with $2 \times 40 \mathrm{ml}$ of $\mathrm{CH}_{2} \mathrm{Cl}_{2}$ and drying in vacuo gave $21.22 \mathrm{~g}(99.8 \%)$ of $\left[\mathrm{R}_{\mathrm{F} 8}\left(\mathrm{CH}_{2}\right)_{3} \mathrm{P}\left(\mathrm{CH}_{2} \mathrm{CH}_{2} \mathrm{CN}\right)_{3}\right]^{+} \mathrm{I}^{-}$as a white powder. $21.00 \mathrm{~g}(26.88 \mathrm{mmol})$ of this salt was weighted into a heavy-walled roundbottomed flask and treated with $\mathrm{NaOMe}(30 \mathrm{w} / \mathrm{w} \%$ solution in methanol, $9.68 \mathrm{~g}, 53.76$ mmol) and $\mathrm{MeOH}(28 \mathrm{ml},<0.1 \%$ water). The flask was closed with a stop-cock, clamped, and heated to $70^{\circ} \mathrm{C}$ with vigorous stirring. The initial suspension turned into solution after about 30 minutes. After 12 hours the reaction mixture was evaporated to dryness at room temperature using rotary evaporator combined with water-vacuo. The brown residue was then suspended in water $(100 \mathrm{ml})$ and filtered through a Büchnerfunnel. The remaining solid was suspended again in $50 \mathrm{ml}$ of water, filtered, dissolved in $250 \mathrm{ml}$ of $\mathrm{CHCl}_{3}: \mathrm{CH}_{3} \mathrm{OH}(10: 1)$ and dried over desiccated $\mathrm{MgSO}_{4}$. The solution was then filtered, evaporated to dryness and the residue was finally dried in vacuo $\left(80^{\circ} \mathrm{C}, 0.05\right.$ mbar) giving $13.19 \mathrm{~g}(81.8 \%)$ of $\mathrm{R}_{8}\left(\mathrm{CH}_{2}\right)_{3} \mathrm{P}\left(\mathrm{CH}_{2} \mathrm{CH}_{2} \mathrm{CN}\right)_{2}$ as a white powder. The alkylation of this phosphine $(12.40 \mathrm{~g}, 20.66 \mathrm{mmol})$ was carried out similarly to the first alkylation step with (18.22 g, $30.98 \mathrm{mmol})$ of $\mathrm{R}_{\mathrm{F} 8}\left(\mathrm{CH}_{2}\right)_{3} \mathrm{I}$ and resulted in $24.06 \mathrm{~g}(98.0 \%)$ of $\left\{\left[\mathrm{R}_{88}\left(\mathrm{CH}_{2}\right)_{3}\right]_{2} \mathrm{P}\left(\mathrm{CH}_{2} \mathrm{CH}_{2} \mathrm{CN}\right)_{2}\right\}^{+} \mathrm{I}^{-}$as a white powder. Dealkylation was performed with $(20.00 \mathrm{~g}, 16.83 \mathrm{mmol})$ of this salt and $(6.06 \mathrm{~g}, 33.66 \mathrm{mmol})$ of a NaOMe-solution in $18 \mathrm{ml}$ of $\mathrm{MeOH}: \mathrm{EtOH}(2: 1)$ at $80^{\circ} \mathrm{C}$. The same work-up procedure as used for the previous dealkylation gave $13.40 \mathrm{~g}(79.1 \%)$ of $\left[\mathrm{R}_{\mathrm{F} 8}\left(\mathrm{CH}_{2}\right)_{3}\right]_{2} \mathrm{PCH}_{2} \mathrm{CH}_{2} \mathrm{CN}$ as an off-white powder. This phosphine showed no air-sensitivity after a week at room-temperature. Next, $14.60 \mathrm{~g}(87.8 \%)$ of $\left\{\left[\mathrm{R}_{\mathrm{F} 8}\left(\mathrm{CH}_{2}\right)_{3}\right]_{3} \quad \mathrm{PCH}_{2} \mathrm{CH}_{2} \mathrm{CN}\right\}^{+} \mathrm{I}^{-}$was obtained from this phosphine (10.50 g, $10.42 \mathrm{mmol})$ and $\mathrm{R}_{\mathrm{F} 8}\left(\mathrm{CH}_{2}\right)_{3} \mathrm{I}(12.26 \mathrm{~g}, 20.84 \mathrm{mmol})$ applying the general alkylation procedure, after purification by flash chromatography on alumina and 
a small amount of carbon-black (eluted with acetone). This chromatography is needed to get out the traces of inpurities, which are difficult to be removed from the final fluorous phosphine. During the last step, all manipulations were performed strictly under nitrogen. This salt (14.00 g, $8.775 \mathrm{mmol})$ was dealkylated with (3.16 g, $17.55 \mathrm{mmol})$ of a NaOMesolution in $14 \mathrm{ml}$ of $\mathrm{MeOH}: \mathrm{EtOH}(1: 1)$ at $80^{\circ} \mathrm{C}$ for 14 hours in the general way. After cooling to room temperature $\mathrm{O}_{2}$-free methanol $(25 \mathrm{ml})$ and $\mathrm{FC}-72(40 \mathrm{ml})$ was added to the mixture. The extraction at $30^{\circ} \mathrm{C}$ was followed by further $40 \mathrm{ml}$ of $\mathrm{O}_{2}$-free FC-72. All fluorous phases were combined and the solution was evaporated to dryness at atmospheric pressure. Drying in high-vacuum at $80^{\circ} \mathrm{C}$ gave $12.05 \mathrm{~g}(97.1 \%)$ of $\left[\mathrm{R}_{\mathrm{F}}\left(\mathrm{CH}_{2}\right)_{3}\right]_{3} \mathrm{P}$ as an off-white powder.

In the case of $\left[\mathrm{R}_{\mathrm{F} 4}\left(\mathrm{CH}_{2}\right)_{3}\right]_{3} \mathrm{P}$ and $\left[\mathrm{R}_{\mathrm{F} 6}\left(\mathrm{CH}_{2}\right)_{3}\right]_{3} \mathrm{P}$, the bis-perfluoroalkyl-phosphine intermediates were liquids, while in the case of $\left[\mathrm{R}_{\mathrm{F} 4}\left(\mathrm{CH}_{2}\right)_{3}\right]_{3} \mathrm{P}$ the mono perfluoroalkylphosphine was liquid too. The intermediate phosphine, $\left[\mathrm{R}_{\mathrm{F} 6}\left(\mathrm{CH}_{2}\right)_{3}\right]\left[\mathrm{R}_{\mathrm{F} 8}\left(\mathrm{CH}_{2}\right)_{3}\right]$ $\mathrm{P}\left(\mathrm{CH}_{2} \mathrm{CH}_{2} \mathrm{CN}\right)$ was also a liquid. Therefore, the work-up procedure in these cases after the appropriate dealkylation steps was different from the general. The crude reaction mixture was also evaporated to dryness, treated with water, but after the separation of the phases the water was removed and the organic oil was extracted again with water. After the removal of the aqueous phase, the oil was dissolved in chloroform, dried over desiccated $\mathrm{MgSO}_{4}$, filtered, and evaporated to dryness using rotary evaporator. Finally, the residual oil was heated with stirring under vacuo $(0.1 \mathrm{mbar})$ at $60-70^{\circ} \mathrm{C}$ to remove the traces of 2-methoxypropionitrile. The corresponding phosphines were obtained as light yellow or brown oils. 


\section{NMR Data of Prepared Compounds}

$\left[\mathbf{R}_{\mathbf{F 8}}\left(\mathbf{C H}_{\mathbf{2}}\right)_{\mathbf{3}}\right]\left(\mathbf{N C}-\mathbf{C H}_{\mathbf{2}} \mathbf{C H}_{\mathbf{2}}\right)_{\mathbf{3}} \mathbf{P}^{\mathbf{+}} \mathbf{I}^{-}:{ }^{1} \mathrm{H}-\mathrm{NMR}\left(250 \mathrm{MHz},\left(\mathrm{CD}_{3}\right)_{2} \mathrm{CO}\right): \delta$ 2.24-2.36 (m, 2H), 2.51-2.68 (m, 2H), 3.20-3.29 (m, 2H), 3.31-3.40 (m, 6H), 3.40-3.50 (m, 6H). ${ }^{31} \mathrm{P}\left\{{ }^{1} \mathrm{H}\right\}-\mathrm{NMR}\left(101 \mathrm{MHz},\left(\mathrm{CD}_{3}\right)_{2} \mathrm{CO}\right) \delta 38.7$ (s). ${ }^{19} \mathrm{~F}-\mathrm{NMR}\left(235 \mathrm{MHz},\left(\mathrm{CD}_{3}\right)_{2} \mathrm{CO}\right) \delta-82.1$ (t, 3F), -115.1 (m, 2F), -122.6 (m, 2F), -122.9 (m, 2+2F), -123.7 (m, 2F), -124.5 (m, 2F), $-127.2(\mathrm{~m}, 2 \mathrm{~F})$.

$\left[\mathbf{R}_{\mathbf{F 8}}\left(\mathbf{C H}_{2}\right)_{3}\right]\left(\mathbf{N C}-\mathbf{C H}_{2} \mathbf{C H}_{2}\right)_{2} \mathbf{P}:{ }^{1} \mathrm{H}-\mathrm{NMR}\left(250 \mathrm{MHz}, \mathrm{CDCl}_{3}\right): \delta$ 1.64-1.71 (m, $2 \mathrm{H}), 1.76-1.92(\mathrm{~m}, 2+4 \mathrm{H}), 2.15-2.32(\mathrm{~m}, 2 \mathrm{H}), 2.53-2.61(\mathrm{~m}, 4 \mathrm{H}) .{ }^{31} \mathrm{P}\left\{{ }^{1} \mathrm{H}\right\}-\mathrm{NMR}(101$ $\left.\mathrm{MHz}, \mathrm{CDCl}_{3}\right) \delta-24.4(\mathrm{~s}) .{ }^{19} \mathrm{~F}-\mathrm{NMR}\left(235 \mathrm{MHz}, \mathrm{CDCl}_{3}\right) \delta-81.2(\mathrm{t}, 3 \mathrm{~F}),-114.6(\mathrm{~m}, 2 \mathrm{~F})$, 122.1 (m, 2F), -122.3 (m, 2+2F), -123.1 (m, 2F), -123.9 (m, 2F), -126.5 (m, 2F).

$\left[\mathbf{R}_{\mathbf{F 8}}\left(\mathbf{C H}_{2}\right)_{3}\right]_{\mathbf{2}}\left(\mathbf{N C}-\mathbf{C H}_{\mathbf{2}} \mathbf{C H}_{2}\right)_{\mathbf{2}} \mathbf{P}^{\mathbf{+}} \mathbf{I}^{-}:{ }^{1} \mathrm{H}-\mathrm{NMR}\left(250 \mathrm{MHz},\left(\mathrm{CD}_{3}\right)_{2} \mathrm{CO}\right): \delta$ 2.08-2.21 (m, 4H), 2.38-2.55 (m, 4H), 2.98-3.11 (m, 4H), 3.15-3.30 (m, 4+4H). ${ }^{31} \mathrm{P}\left\{{ }^{1} \mathrm{H}\right\}-\mathrm{NMR}(101$ $\left.\mathrm{MHz},\left(\mathrm{CD}_{3}\right)_{2} \mathrm{CO}\right) \delta 37.1(\mathrm{~s}) .{ }^{19} \mathrm{~F}-\mathrm{NMR}\left(235 \mathrm{MHz},\left(\mathrm{CD}_{3}\right)_{2} \mathrm{CO}\right) \delta-82.1(\mathrm{t}, 6 \mathrm{~F}),-115.0(\mathrm{~m}$, 4F), -122.7 (m, 4F), - 122.9 (m, 4+4F), -123.7 (m, 4F), -124.4 (m, 4F), -127.2 (m, 4F).

$\left[\mathbf{R}_{\mathbf{F} 8}\left(\mathbf{C H}_{2}\right)_{3}\right]_{\mathbf{2}}\left(\mathbf{N C}-\mathbf{C H}_{\mathbf{2}} \mathbf{C H}_{\mathbf{2}}\right) \mathbf{P}:{ }^{1} \mathrm{H}-\mathrm{NMR}\left(250 \mathrm{MHz}, \mathrm{CDCl}_{3}: \mathrm{CD}_{3} \mathrm{OD}(9: 1)\right): \delta$ 1.59-1.66 (m, 4H), 1.73-1.85 (m, 2+4H), 2.17-2.35 (m, 4H), 2.55-2.64 (m, 2H). ${ }^{31} \mathrm{P}\left\{{ }^{1} \mathrm{H}\right\}-$ NMR (101 MHz, CDCl $\left.{ }_{3}: \mathrm{CD}_{3} \mathrm{OD}(9: 1)\right) \delta-29.2(\mathrm{~s}) .{ }^{19} \mathrm{~F}-\mathrm{NMR}\left(235 \mathrm{MHz}, \mathrm{CDCl}_{3}: \mathrm{CD}_{3} \mathrm{OD}\right.$ (9:1)) $\delta$-82.1 (t, 6F), -115.1(m, 4F), -122.7 (m, 4F), -122.9 (m, 4+4F), -123.8 (m, 4F), $124.5(\mathrm{~m}, 4 \mathrm{~F}),-127.2(\mathrm{~m}, 4 \mathrm{~F})$.

$\left[\mathbf{R}_{\mathbf{F 8}}\left(\mathbf{C H}_{2}\right)_{3}\right]_{\mathbf{3}}\left(\mathbf{N C}-\mathbf{C H}_{\mathbf{2}} \mathbf{C H}_{2}\right) \mathbf{P}^{+} \mathbf{I}^{-}:{ }^{1} \mathrm{H}-\mathrm{NMR}\left(250 \mathrm{MHz},\left(\mathrm{CD}_{3}\right)_{2} \mathrm{CO}\right): \delta$ 2.04-2.19 (m, 6H), 2.38-2.56 (m, 6H), 2.91-3.03 (m, 6H), 3.12-3.25 (m, 2+2H). ${ }^{31} \mathrm{P}\left\{{ }^{1} \mathrm{H}\right\}-\mathrm{NMR}(101$ $\left.\mathrm{MHz},\left(\mathrm{CD}_{3}\right)_{2} \mathrm{CO}\right) \delta 36.2$ (s). ${ }^{19} \mathrm{~F}-\mathrm{NMR}\left(235 \mathrm{MHz},\left(\mathrm{CD}_{3}\right)_{2} \mathrm{CO}\right) \delta-82.1$ (t, 9F), -114.9 (m, $6 \mathrm{~F}),-122.7(\mathrm{~m}, 6 \mathrm{~F}),-122.9(\mathrm{~m}, 6+6 \mathrm{~F}),-123.8(\mathrm{~m}, 6 \mathrm{~F}),-124.4(\mathrm{~m}, 6 \mathrm{~F}),-127.2(\mathrm{~m}, 6 \mathrm{~F})$.

$\left[\mathbf{R}_{\mathbf{F 8}}\left(\mathbf{C H}_{2}\right)_{3}\right]_{3} \mathbf{P}:{ }^{1} \mathrm{H}-\mathrm{NMR}(250 \mathrm{MHz}, \mathrm{FC}-72): \delta$ 1.50-1.63 (m, 6H), 1.80-2.00 (m, $6 \mathrm{H}), 2.14-2.40(\mathrm{~m}, 6 \mathrm{H}) .{ }^{31} \mathrm{P}\left\{{ }^{1} \mathrm{H}\right\}-\mathrm{NMR}(101 \mathrm{MHz}, \mathrm{FC}-72) \delta-34.4(\mathrm{~s}) .{ }^{19} \mathrm{~F}-\mathrm{NMR}(235$ $\left.\mathrm{MHz},\left(\mathrm{CD}_{3}\right)_{2} \mathrm{CO}\right) \delta-82.2(\mathrm{t}, 9 \mathrm{~F}),-115.2(\mathrm{~m}, 6 \mathrm{~F}),-122.7$ (m, 6F), -122.9 (m, 6+6F), -123.8 $(\mathrm{m}, 6 \mathrm{~F}),-124.5(\mathrm{~m}, 6 \mathrm{~F}),-127.2(\mathrm{~m}, 6 \mathrm{~F})$. 
$\left[\mathbf{R}_{\mathbf{F} 6}\left(\mathbf{C H}_{2}\right)_{3}\right]\left(\mathbf{N C}-\mathbf{C H}_{\mathbf{2}} \mathbf{C H}_{2}\right)_{3} \mathbf{P}^{+} \mathbf{I}^{-}:{ }^{1} \mathrm{H}-\mathrm{NMR}\left(250 \mathrm{MHz},\left(\mathrm{CD}_{3}\right)_{2} \mathrm{CO}\right):$ 2.20-2.38 (m, $2 \mathrm{H}), 2.46-2.72(\mathrm{~m}, 2 \mathrm{H}), 3.17-3.32(\mathrm{~m}, 2 \mathrm{H}), 3.32-3.40(\mathrm{~m}, 6 \mathrm{H}), 3.40-3.53(\mathrm{~m}, 6 \mathrm{H})$. ${ }^{31} \mathrm{P}\left\{{ }^{1} \mathrm{H}\right\}-\mathrm{NMR}\left(101 \mathrm{MHz},\left(\mathrm{CD}_{3}\right)_{2} \mathrm{CO}\right) \delta 38.6(\mathrm{~s})$.

$\left[\mathbf{R}_{\mathbf{F} 6}\left(\mathbf{C H}_{2}\right)_{3}\right]\left(\mathbf{N C}-\mathbf{C H}_{2} \mathbf{C H}_{2}\right)_{2} \mathbf{P}:{ }^{1} \mathrm{H}-\mathrm{NMR}\left(250 \mathrm{MHz}, \mathrm{CDCl}_{3}\right): \delta$ 1.59-1.69 (m, 2H), 1.71-1.91 (m, 2+4H), 2.09-2.33 (m, 2H), 2.48-2.62 (m, 4H). ${ }^{31} \mathrm{P}\left\{{ }^{1} \mathrm{H}\right\}-\mathrm{NMR}(101$ $\left.\mathrm{MHz}, \mathrm{CDCl}_{3}\right) \delta-23.6(\mathrm{~s})$.

$\left[\mathbf{R}_{\mathbf{F} 6}\left(\mathbf{C H}_{2}\right)_{3}\right]_{\mathbf{2}}\left(\mathbf{N C}-\mathbf{C H}_{\mathbf{2}} \mathbf{C H}_{2}\right)_{\mathbf{2}} \mathbf{P}^{\mathbf{+}} \mathbf{I}^{-}:{ }^{1} \mathrm{H}-\mathrm{NMR}\left(250 \mathrm{MHz},\left(\mathrm{CD}_{3}\right)_{2} \mathrm{CO}\right): \delta$ 2.16-2.35 (m, 4H), 2.46-2.71 (m, 4H), 3.09-3.25 (m, 4H), 3.26-3.45 (m, 4+4H). ${ }^{31} \mathrm{P}\left\{{ }^{1} \mathrm{H}\right\}-\mathrm{NMR}(101$ $\left.\mathrm{MHz},\left(\mathrm{CD}_{3}\right)_{2} \mathrm{CO}\right) \delta 37.8(\mathrm{~s}) .{ }^{19} \mathrm{~F}-\mathrm{NMR}\left(235 \mathrm{MHz},\left(\mathrm{CD}_{3}\right)_{2} \mathrm{CO}\right) \delta-82.2(\mathrm{t}, 6 \mathrm{~F}),-115.0(\mathrm{~m}$, 4F), -122.9 (m, 4F), -123.9 (m, 4F), -124.5 (m, 4F), -127.2 (m, 4F).

$\left[\mathbf{R}_{\mathbf{F} 6}\left(\mathbf{C H}_{2}\right)_{3}\right]_{\mathbf{2}}\left(\mathbf{N C}-\mathbf{C H}_{\mathbf{2}} \mathbf{C H}_{2}\right) \mathbf{P}:{ }^{1} \mathrm{H}-\mathrm{NMR}\left(250 \mathrm{MHz}, \mathrm{CDCl}_{3}\right): \delta$ 1.51-1.62 (m, $4 \mathrm{H}), 1.68-1.87(\mathrm{~m}, 2+4 \mathrm{H}), 2.07-2.33(\mathrm{~m}, 4 \mathrm{H}), 2.44-2.57(\mathrm{~m}, 2 \mathrm{H}) .{ }^{31} \mathrm{P}\left\{{ }^{1} \mathrm{H}\right\}-\mathrm{NMR}(101$ $\left.\mathrm{MHz}, \mathrm{CDCl}_{3}\right) \delta-27.4(\mathrm{~s})$.

$\left[\mathbf{R}_{\mathbf{F} 6}\left(\mathbf{C H}_{2}\right)_{3}\right]_{\mathbf{3}}\left(\mathbf{N C}-\mathbf{C H}_{\mathbf{2}} \mathbf{C H}_{2}\right) \mathbf{P}^{+} \mathbf{I}^{-}:{ }^{1} \mathrm{H}-\mathrm{NMR}\left(250 \mathrm{MHz},\left(\mathrm{CD}_{3}\right)_{2} \mathrm{CO}\right): \delta$ 2.12-2.32 $(\mathrm{m}, 6 \mathrm{H}), 2.46-2.71(\mathrm{~m}, 6 \mathrm{H}), 3.02-3.19(\mathrm{~m}, 6 \mathrm{H}), 3.22-3.46(\mathrm{~m}, 2+2 \mathrm{H}) .{ }^{31} \mathrm{P}\left\{{ }^{1} \mathrm{H}\right\}-\mathrm{NMR}(101$ $\left.\mathrm{MHz},\left(\mathrm{CD}_{3}\right)_{2} \mathrm{CO}\right) \delta 37.0(\mathrm{~s})$.

$\left[\mathbf{R}_{\mathbf{F} 6}\left(\mathbf{C H}_{2}\right)_{3}\right]_{3} \mathbf{P}:{ }^{1} \mathrm{H}-\mathrm{NMR}(250 \mathrm{MHz}, \mathrm{FC}-72): \delta$ 1.52-1.63 (m, 6H), 1.82-2.01 (m, $6 \mathrm{H}), 2.16-2.41(\mathrm{~m}, 6 \mathrm{H}) .{ }^{31} \mathrm{P}\left\{{ }^{1} \mathrm{H}\right\}-\mathrm{NMR}(101 \mathrm{MHz}, \mathrm{FC}-72) \delta-36.0(\mathrm{~s})$.

$\left[\mathbf{R}_{\mathbf{F} 4}\left(\mathbf{C H}_{2}\right)_{3}\right]\left(\mathbf{N C}-\mathbf{C H}_{\mathbf{2}} \mathbf{C H}_{2}\right)_{3} \mathbf{P}^{+} \mathbf{I}^{-}:{ }^{1} \mathrm{H}-\mathrm{NMR}\left(250 \mathrm{MHz},\left(\mathrm{CD}_{3}\right)_{2} \mathrm{CO}\right): \delta$ 2.18-2.36 (m, 2H), 2.45-2.70 (m, 2H), 3.14-3.29 (m, 2H), 3.29-3.39 (m, 6H), 3.39-3.52 (m, 6H). ${ }^{31} \mathrm{P}\left\{{ }^{1} \mathrm{H}\right\}-\mathrm{NMR}\left(101 \mathrm{MHz},\left(\mathrm{CD}_{3}\right)_{2} \mathrm{CO}\right) \delta 38.6(\mathrm{~s}) .{ }^{19} \mathrm{~F}-\mathrm{NMR}\left(235 \mathrm{MHz},\left(\mathrm{CD}_{3}\right)_{2} \mathrm{CO}\right) \delta-82.4$ (t, 3F), -115.3 (m, 2F), -125.5 (m, 2F), -127.1 (m, 2F).

$\left[\mathbf{R}_{\mathbf{F} 4}\left(\mathbf{C H}_{2}\right)_{3}\right]\left(\mathbf{N C}-\mathbf{C H}_{\mathbf{2}} \mathbf{C H}_{2}\right)_{2} \mathbf{P}:{ }^{1} \mathrm{H}-\mathrm{NMR}\left(250 \mathrm{MHz}, \mathrm{CDCl}_{3}\right): \delta$ 1.59-1.68 (m, 2H), 1.69-1.89 (m, 2+4H), 2.08-2.33 (m, 2H), 2.47-2.61 (m, 4H). ${ }^{31} \mathrm{P}\left\{{ }^{1} \mathrm{H}\right\}-\mathrm{NMR}(101$ $\left.\mathrm{MHz}, \mathrm{CDCl}_{3}\right) \delta-23.7(\mathrm{~s})$.

$\left[\mathbf{R}_{\mathbf{F} 4}\left(\mathbf{C H}_{2}\right)_{3}\right]_{\mathbf{2}}\left(\mathbf{N C}-\mathbf{C H}_{\mathbf{2}} \mathbf{C H}_{2}\right)_{\mathbf{2}} \mathbf{P}^{\mathbf{+}} \mathbf{I}^{-}:{ }^{1} \mathrm{H}-\mathrm{NMR}\left(250 \mathrm{MHz},\left(\mathrm{CD}_{3}\right)_{2} \mathrm{CO}\right): \delta$ 2.15-2.35 (m, 4H), 2.45-2.71 (m, 4H), 3.07-3.23 (m, 4H), 3.26-3.44 (m, 4+4H). ${ }^{31} \mathrm{P}\left\{{ }^{1} \mathrm{H}\right\}-\mathrm{NMR}(101$ $\left.\mathrm{MHz},\left(\mathrm{CD}_{3}\right)_{2} \mathrm{CO}\right) \delta 37.8(\mathrm{~s}) .{ }^{19} \mathrm{~F}-\mathrm{NMR}\left(235 \mathrm{MHz},\left(\mathrm{CD}_{3}\right)_{2} \mathrm{CO}\right) \delta-82.4(\mathrm{t}, 6 \mathrm{~F}),-115.3(\mathrm{~m}$, 4F), -125.5 (m, 4F), - 127.2 (m, 4F). 
$\left[\mathbf{R}_{\mathbf{F} 4}\left(\mathbf{C H}_{2}\right)_{3}\right]_{\mathbf{2}}\left(\mathbf{N C}-\mathbf{C H}_{\mathbf{2}} \mathbf{C H}_{2}\right) \mathbf{P}:{ }^{1} \mathrm{H}-\mathrm{NMR}\left(250 \mathrm{MHz}, \mathrm{CDCl}_{3}\right): \delta$ 1.51-1.61 (m, $4 \mathrm{H}), 1.66-1.86(\mathrm{~m}, 2+4 \mathrm{H}), 2.07-2.30(\mathrm{~m}, 4 \mathrm{H}), 2.44-2.56(\mathrm{~m}, 2 \mathrm{H}) .{ }^{31} \mathrm{P}\left\{{ }^{1} \mathrm{H}\right\}-\mathrm{NMR}(101$ $\left.\mathrm{MHz}, \mathrm{CDCl}_{3}\right) \delta-27.5(\mathrm{~s})$.

$\left[\mathbf{R}_{\mathbf{F}}\left(\mathbf{C H}_{2}\right)_{3}\right]_{\mathbf{3}}\left(\mathbf{N C}-\mathbf{C H}_{\mathbf{2}} \mathbf{C H}_{2}\right) \mathbf{P}^{+} \mathbf{I}^{-}:{ }^{1} \mathrm{H}-\mathrm{NMR}\left(250 \mathrm{MHz},\left(\mathrm{CD}_{3}\right)_{2} \mathrm{CO}\right): \delta$ 2.14-2.32 (m, 6H), 2.45-2.70 (m, 6H), 3.01-3.17 (m, 6H), 3.21-3.42 (m, 2+2H). ${ }^{31} \mathrm{P}\left\{{ }^{1} \mathrm{H}\right\}-\mathrm{NMR}(101$ $\left.\mathrm{MHz},\left(\mathrm{CD}_{3}\right)_{2} \mathrm{CO}\right) \delta 37.0(\mathrm{~s})$.

$\left[\mathbf{R}_{\mathbf{F} 4}\left(\mathbf{C H}_{2}\right)_{3}\right]_{3} \mathbf{P}:{ }^{1} \mathrm{H}-\mathrm{NMR}(250 \mathrm{MHz}, \mathrm{FC}-72): \delta$ 1.51-1.64 (m, 6H), 1.81-2.01 (m, $6 \mathrm{H}), 2.15-2.40(\mathrm{~m}, 6 \mathrm{H}) .{ }^{31} \mathrm{P}\left\{{ }^{1} \mathrm{H}\right\}-\mathrm{NMR}$ (101 MHz, FC-72) $\delta$-34.5 (s).

$\left[\mathbf{R}_{\mathbf{F 8}}\left(\mathbf{C H}_{2}\right)_{4}\right]\left(\mathbf{N C}-\mathbf{C H}_{\mathbf{2}} \mathbf{C H}_{2}\right)_{3} \mathbf{P}^{+} \mathbf{I}^{-}:{ }^{1} \mathrm{H}-\mathrm{NMR}\left(250 \mathrm{MHz},\left(\mathrm{CD}_{3}\right)_{2} \mathrm{CO}\right): \delta$ 1.83-1.99 $(\mathrm{m}, 2 \mathrm{H}), 2.03-2.21(\mathrm{~m}, 2 \mathrm{H}), 2.26-2.52(\mathrm{~m}, 2 \mathrm{H}), 3.04-3.19(\mathrm{~m}, 2 \mathrm{H}), 3.25-3.36(\mathrm{~m}, 6 \mathrm{H})$, 3.36-3.48 (m, 6H). ${ }^{31} \mathrm{P}\left\{{ }^{1} \mathrm{H}\right\}-\mathrm{NMR}\left(101 \mathrm{MHz},\left(\mathrm{CD}_{3}\right)_{2} \mathrm{CO}\right) \delta 38.4(\mathrm{~s})$.

$\left[\mathbf{R}_{\mathbf{F 8}}\left(\mathbf{C H}_{2}\right)_{4}\right]\left(\mathbf{N C}-\mathbf{C H}_{\mathbf{2}} \mathbf{C H}_{2}\right)_{2} \mathbf{P}:{ }^{1} \mathrm{H}-\mathrm{NMR}\left(250 \mathrm{MHz}, \mathrm{CDCl}_{3}\right): \delta$ 1.52-1.62 (m, $2+2 \mathrm{H}), 1.67-1.79(\mathrm{~m}, 2 \mathrm{H}), 1.78-1.89(\mathrm{t}, 4 \mathrm{H}), 1.99-2.24(\mathrm{~m}, 2 \mathrm{H}), 2.46-2.60(\mathrm{~m}, 4 \mathrm{H})$. ${ }^{31} \mathrm{P}\left\{{ }^{1} \mathrm{H}\right\}-\mathrm{NMR}\left(101 \mathrm{MHz}, \mathrm{CDCl}_{3}\right) \delta-23.3(\mathrm{~s})$.

$\left[\mathbf{R}_{\mathbf{F 8}}\left(\mathbf{C H}_{2}\right)_{\mathbf{4}}\right]_{\mathbf{2}}\left(\mathbf{N C}-\mathbf{C H}_{\mathbf{2}} \mathbf{C H}_{2}\right)_{\mathbf{2}} \mathbf{P}^{\mathbf{+}} \mathbf{I}^{-}:{ }^{1} \mathrm{H}-\mathrm{NMR}\left(250 \mathrm{MHz},\left(\mathrm{CD}_{3}\right)_{2} \mathrm{CO}\right): \delta$ 1.82-1.97 (m, 4H), 1.99-2.17 (m, 4H), 2.25-2.51 (m, 4H), 2.91-3.07 (m, 4H), 3.18-3.36 (m, 4+4H). ${ }^{31} \mathrm{P}\left\{{ }^{1} \mathrm{H}\right\}-\mathrm{NMR}\left(101 \mathrm{MHz},\left(\mathrm{CD}_{3}\right)_{2} \mathrm{CO}\right) \delta 37.5(\mathrm{~s})$.

$\left[\mathbf{R}_{\mathbf{F 8}}\left(\mathbf{C H}_{2}\right)_{4}\right]_{\mathbf{2}}\left(\mathbf{N C}-\mathbf{C H}_{2} \mathbf{C H}_{2}\right) \mathbf{P}:{ }^{1} \mathrm{H}-\mathrm{NMR}\left(250 \mathrm{MHz}, \mathrm{CDCl}_{3}\right): \delta$ 1.39-1.64 (m, $4+4 \mathrm{H}), 1.64-1.82(\mathrm{~m}, 4+2 \mathrm{H}), 1.95-2.23(\mathrm{~m}, 4 \mathrm{H}), 2.40-2.55(\mathrm{~m}, 2 \mathrm{H}) .{ }^{31} \mathrm{P}\left\{{ }^{1} \mathrm{H}\right\}-\mathrm{NMR}(101$ $\left.\mathrm{MHz}, \mathrm{CDCl}_{3}\right) \delta-26.6(\mathrm{~s})$.

$\left[\mathbf{R}_{\mathbf{F 8}}\left(\mathbf{C H}_{2}\right)_{\mathbf{4}}\right]_{\mathbf{3}}\left(\mathbf{N C}-\mathbf{C H}_{\mathbf{2}} \mathbf{C H}_{2}\right) \mathbf{P}^{+} \mathbf{I}^{-}:{ }^{1} \mathrm{H}-\mathrm{NMR}\left(250 \mathrm{MHz},\left(\mathrm{CD}_{3}\right)_{2} \mathrm{CO}\right): \delta$ 1.82-1.95 (m, 6H), 1.96-2.11 (m, 6H), 2.25-2.50 (m, 6H), 2.76-2.94 (m, 6H), 3.06-3.31 (m, 2+2H). ${ }^{31} \mathrm{P}\left\{{ }^{1} \mathrm{H}\right\}-\mathrm{NMR}\left(101 \mathrm{MHz},\left(\mathrm{CD}_{3}\right)_{2} \mathrm{CO}\right) \delta 36.5(\mathrm{~s})$.

$\left[\mathbf{R}_{\mathbf{F 8}}\left(\mathbf{C H}_{2}\right)_{4}\right]_{3} \mathbf{P}:{ }^{1} \mathrm{H}-\mathrm{NMR}(250 \mathrm{MHz}, \mathrm{FC}-72): \delta$ 1.36-1.49 (m, 6H), 1.49-1.67 (m, $6 \mathrm{H}), 1.67-1.87(\mathrm{~m}, 6 \mathrm{H}), 1.93-2.23(\mathrm{~m}, 6 \mathrm{H}) .{ }^{31} \mathrm{P}\left\{{ }^{1} \mathrm{H}\right\}-\mathrm{NMR}(101 \mathrm{MHz}, \mathrm{FC}-72) \delta-32.4(\mathrm{~s})$.

$\left[\mathbf{R}_{\mathbf{F} 8}\left(\mathbf{C H}_{2}\right)_{3}\right]\left(\mathbf{N C}-\mathbf{C H}_{\mathbf{2}} \mathbf{C H}_{2}\right)_{3} \mathbf{P}^{+} \mathbf{I}^{-}:{ }^{1} \mathrm{H}-\mathrm{NMR}\left(250 \mathrm{MHz},\left(\mathrm{CD}_{3}\right)_{2} \mathrm{CO}\right): \delta$ 2.24-2.36 (m, 2H), 2.51-2.68 (m, 2H), 3.20-3.29 (m, 2H), 3.31-3.40 (m, 6H), 3.40-3.50 (m, 6H). ${ }^{31} \mathrm{P}\left\{{ }^{1} \mathrm{H}\right\}$-NMR $\left(101 \mathrm{MHz},\left(\mathrm{CD}_{3}\right)_{2} \mathrm{CO}\right) \delta 38.7$ (s). 
$\left[\mathbf{R}_{\mathbf{F} 8}\left(\mathbf{C H}_{2}\right)_{3}\right]\left(\mathbf{N C}-\mathbf{C H}_{2} \mathbf{C H}_{2}\right)_{2} \mathbf{P}:{ }^{1} \mathrm{H}-\mathrm{NMR}\left(250 \mathrm{MHz},\left(\mathrm{CD}_{3}\right)_{2} \mathrm{CO}\right): \delta$ 1.64-1.71 (m, 2H), 1.76-1.92 (m, 2+4H), 2.15-2.32 (m, 2H), 2.53-2.61 (m, 4H). ${ }^{31} \mathrm{P}\left\{{ }^{1} \mathrm{H}\right\}-\mathrm{NMR}(101$ $\left.\mathrm{MHz},\left(\mathrm{CD}_{3}\right)_{2} \mathrm{CO}\right) \delta-24.4(\mathrm{~s})$.

$\left[\mathbf{R}_{\mathbf{F 8}}\left(\mathbf{C H}_{2}\right)_{3}\right]\left[\mathbf{R}_{\mathbf{F 8}}\left(\mathbf{C H}_{2}\right)_{\mathbf{4}}\right]\left(\mathbf{N C}-\mathbf{C H}_{\mathbf{2}} \mathbf{C H}_{2}\right)_{2} \mathbf{P}^{+} \mathbf{I}^{-}:{ }^{1} \mathrm{H}-\mathrm{NMR}\left(250 \mathrm{MHz},\left(\mathrm{CD}_{3}\right)_{2} \mathrm{CO}\right): \delta$ 1.85-1.97 (m, 2H), 1.98-2.70 (m, 2+2+2+2H), 2.95-3.19 (m, 2+2H), 3.20-3.40 (m, $4+4 \mathrm{H}) .{ }^{31} \mathrm{P}\left\{{ }^{1} \mathrm{H}\right\}-\mathrm{NMR}\left(101 \mathrm{MHz},\left(\mathrm{CD}_{3}\right)_{2} \mathrm{CO}\right) \delta 37.7$ (s).

$\left[\mathbf{R}_{\mathbf{F 8}}\left(\mathbf{C H}_{2}\right)_{3}\right]\left[\mathbf{R}_{\mathbf{F 8}}\left(\mathbf{C H}_{2}\right)_{\mathbf{4}}\right]\left(\mathbf{N C}-\mathbf{C H}_{\mathbf{2}} \mathbf{C H}_{2}\right) \mathbf{P}:{ }^{1} \mathrm{H}-\mathrm{NMR}\left(250 \mathrm{MHz},\left(\mathrm{CD}_{3}\right)_{2} \mathrm{CO}\right): \delta$ 1.45-1.64 (m, 2+2+2H), 1.65-1.87 (m, 2+2+2H), 2.96-2.32 (m, 2+2H), 2.43-2.57 (m, 2H). ${ }^{31} \mathrm{P}\left\{{ }^{1} \mathrm{H}\right\}-\mathrm{NMR}\left(101 \mathrm{MHz},\left(\mathrm{CD}_{3}\right)_{2} \mathrm{CO}\right) \delta-27.1$ (s).

$\left[\mathbf{R}_{\mathbf{F 8}}\left(\mathbf{C H}_{2}\right)_{3}\right]\left[\mathbf{R}_{\mathbf{F 8}}\left(\mathbf{C H}_{2}\right)_{\mathbf{4}}\right]_{\mathbf{2}}\left(\mathbf{N C}-\mathbf{C H}_{\mathbf{2}} \mathbf{C H}_{2}\right) \mathbf{P}^{+} \mathbf{I}^{-}:{ }^{1} \mathrm{H}-\mathrm{NMR}\left(250 \mathrm{MHz},\left(\mathrm{CD}_{3}\right)_{2} \mathrm{CO}\right): \delta$ 1.83-1.97 (m, 4H), 1.97-2.70 (m, 2+2+4+4H), 2.73-3.07 (m, 2+4H), 3.11-3.34 (m, $2+2 \mathrm{H}) .{ }^{31} \mathrm{P}\left\{{ }^{1} \mathrm{H}\right\}-\mathrm{NMR}\left(101 \mathrm{MHz},\left(\mathrm{CD}_{3}\right)_{2} \mathrm{CO}\right) \delta 36.8(\mathrm{~s})$.

$\left[\mathbf{R}_{\mathbf{F 8}}\left(\mathbf{C H}_{2}\right)_{3}\right]\left[\mathbf{R}_{\mathbf{F r}}\left(\mathbf{C H}_{2}\right)_{4}\right]_{2} \mathbf{P}:{ }^{1} \mathrm{H}-\mathrm{NMR}(250 \mathrm{MHz}, \mathrm{FC}-72): \delta$ 1.39-1.52 (m, 2+4H), 1.52-1.68 (m, 4H), 1.69-1.92 (m, 2+4H), 1.97-2.33 (m, 2+4H). ${ }^{31} \mathrm{P}\left\{{ }^{1} \mathrm{H}\right\}-\mathrm{NMR}$ (101 MHz, FC-72) $\delta-34.7$ (s).

$\left[\mathbf{R}_{\mathbf{F} 6}\left(\mathbf{C H}_{2}\right)_{3}\right]\left(\mathbf{N C}-\mathbf{C H}_{\mathbf{2}} \mathbf{C H}_{2}\right)_{3} \mathbf{P}^{+} \mathbf{I}^{-}:{ }^{1} \mathrm{H}-\mathrm{NMR}\left(250 \mathrm{MHz},\left(\mathrm{CD}_{3}\right)_{2} \mathrm{CO}\right): \delta$ 2.20-2.38 (m, 2H), 2.46-2.72 (m, 2H), 3.17-3.32 (m, 2H), 3.32-3.40 (m, 6H), 3.40-3.53 (m, 6H). ${ }^{31} \mathrm{P}\left\{{ }^{1} \mathrm{H}\right\}-\mathrm{NMR}\left(101 \mathrm{MHz},\left(\mathrm{CD}_{3}\right)_{2} \mathrm{CO}\right) \delta 38.6(\mathrm{~s})$.

$\left[\mathbf{R}_{\mathbf{F} 6}\left(\mathbf{C H}_{2}\right)_{3}\right]\left(\mathbf{N C}-\mathbf{C H}_{\mathbf{2}} \mathbf{C H}_{2}\right)_{2} \mathbf{P}:{ }^{1} \mathrm{H}-\mathrm{NMR}\left(250 \mathrm{MHz},\left(\mathrm{CD}_{3}\right)_{2} \mathrm{CO}\right): \delta$ 1.59-1.69 (m, 2H), 1.71-1.91 (m, 2+4H), 2.09-2.33 (m, 2H), 2.48-2.62 (m, 4H). ${ }^{31} \mathrm{P}\left\{{ }^{1} \mathrm{H}\right\}-\mathrm{NMR}(101$ $\left.\mathrm{MHz},\left(\mathrm{CD}_{3}\right)_{2} \mathrm{CO}\right) \delta-23.6(\mathrm{~s})$.

$\left[\mathbf{R}_{\mathbf{F} 6}\left(\mathbf{C H}_{2}\right)_{3}\right]\left[\mathbf{R}_{\mathbf{F 8}}\left(\mathbf{C H}_{2}\right)_{3}\right]\left(\mathbf{N C}-\mathbf{C H}_{2} \mathbf{C H}_{2}\right)_{2} \mathbf{P}^{+} \mathbf{I}^{-}:{ }^{1} \mathrm{H}-\mathrm{NMR}\left(250 \mathrm{MHz},\left(\mathrm{CD}_{3}\right)_{2} \mathrm{CO}\right): \delta$ 2.16-2.35 (m, 2+2H), 2.47-2.72 (m, 2+2H), 3.09-3.25 (m, 2+2H), 3.26-3.46 (m, 4+4H). ${ }^{31} \mathrm{P}\left\{{ }^{1} \mathrm{H}\right\}-\mathrm{NMR}\left(101 \mathrm{MHz},\left(\mathrm{CD}_{3}\right)_{2} \mathrm{CO}\right) \delta 37.8(\mathrm{~s}) .{ }^{19} \mathrm{~F}-\mathrm{NMR}\left(235 \mathrm{MHz},\left(\mathrm{CD}_{3}\right)_{2} \mathrm{CO}\right) \delta$ 82.1-(-82.3) (m, 3+3F), -114.9-(-115.1) (m, 2+2F), -122.5-(-123.1) (m 2+2+2+2F), 123.6-(-124.1) (m, 2+2F), -124.3-(-124.7) (m, 2+2F), -127.1-(-127.4) (m, 2+2F).

$\left[\mathbf{R}_{\mathbf{F 6}}\left(\mathbf{C H}_{2}\right)_{3}\right]\left[\mathbf{R}_{\mathbf{H 8}}\left(\mathbf{C H}_{2}\right)_{3}\right]\left(\mathbf{N C}-\mathbf{C H}_{2} \mathbf{C H}_{2}\right) \mathbf{P}:{ }^{1} \mathrm{H}-\mathrm{NMR}\left(250 \mathrm{MHz},\left(\mathrm{CD}_{3}\right)_{2} \mathrm{CO}\right): \delta$ 1.52-1.63 (m, 2+2H), 1.70-1.87 (m, 2+2+2H), 2.07-2.33 (m, 2+2H), 2.44-2.57 (m, 2H). ${ }^{31} \mathrm{P}\left\{{ }^{1} \mathrm{H}\right\}-\mathrm{NMR}\left(101 \mathrm{MHz},\left(\mathrm{CD}_{3}\right)_{2} \mathrm{CO}\right) \delta-27.5$ (s). 
$\left[\mathbf{R}_{\mathbf{F 6}}\left(\mathbf{C H}_{2}\right)_{3}\right]\left[\mathbf{R}_{\mathbf{F 8}}\left(\mathbf{C H}_{2}\right)_{3}\right]\left[\mathbf{R}_{\mathbf{F 8}}\left(\mathbf{C H}_{2}\right)_{4}\right]\left(\mathbf{N C}-\mathbf{C H}_{2} \mathbf{C H}_{2}\right) \mathbf{P}^{+} \mathbf{I}^{-}:{ }^{1} \mathrm{H}-\mathrm{NMR}(250 \mathrm{MHz}$, $\left.\left(\mathrm{CD}_{3}\right)_{2} \mathrm{CO}\right): \delta$ 1.82-1.98 (m, 2H), 2.00-2.72 (m, 2+2+2+2+2+2H), 2.89-3.18 (m, $2+2+2 \mathrm{H}), 3.18-3.45(\mathrm{~m}, 2+2 \mathrm{H}) .{ }^{31} \mathrm{P}\left\{{ }^{1} \mathrm{H}\right\}-\mathrm{NMR}\left(101 \mathrm{MHz},\left(\mathrm{CD}_{3}\right)_{2} \mathrm{CO}\right) \delta 36.8(\mathrm{~s}) .{ }^{19} \mathrm{~F}-$ NMR (235 MHz, $\left.\left(\mathrm{CD}_{3}\right)_{2} \mathrm{CO}\right) \delta$-82.2-(-82.4) (m, 3+3+3F), -114.8-(-115.3) (m, 2+2+2F), -122.6-(-123.2) (m, 2+2+2+2+2+2+2F), -123.6-(-124.2) (m, 2+2+2F), -124.3-(-124.7) $(\mathrm{m}, 2+2+2 \mathrm{~F}),-127.2-(-127.5)(\mathrm{m}, 2+2+2 \mathrm{~F})$.

$\left[\mathbf{R}_{\mathbf{F} 6}\left(\mathbf{C H}_{2}\right)_{3}\right]\left[\mathbf{R}_{\mathbf{F 8}}\left(\mathbf{C H}_{2}\right)_{3}\right]\left[\mathbf{R}_{\mathbf{F 8}}\left(\mathbf{C H}_{2}\right)_{4}\right] \mathbf{P}:{ }^{1} \mathrm{H}-\mathrm{NMR}(250 \mathrm{MHz}, \mathrm{FC}-72): \delta$ 1.46-1.75 $(\mathrm{m}, 2+2+2+2 \mathrm{H}), 1.75-2.00(\mathrm{~m}, 2+2+2 \mathrm{H}), 2.02-2.54(\mathrm{~m}, 2+2+2 \mathrm{H}) .{ }^{31} \mathrm{P}\left\{{ }^{1} \mathrm{H}\right\}-\mathrm{NMR}(101$ $\mathrm{MHz}, \mathrm{FC}-72) \delta-34.0$ (s). ${ }^{19} \mathrm{~F}-\mathrm{NMR}\left(235 \mathrm{MHz},\left(\mathrm{CD}_{3}\right)_{2} \mathrm{CO}\right) \delta-82.1-(-82.3)(\mathrm{m}, 3+3+3 \mathrm{~F}),-$ 114.9-(-115.2) (m, 2+2+2F), -122.6-(-123.2) (m, 2+2+2+2+2+2+2F), -123.6-(-124.2) (m, $2+2+2 \mathrm{~F}),-124.4-(-124.8)(\mathrm{m}, 2+2+2 \mathrm{~F}),-127.1-(-127.5)(\mathrm{m}, 2+2+2 \mathrm{~F})$. 


\section{${ }^{31} \mathbf{P}\left\{{ }^{1} \mathbf{H}\right\}$-NMR Spectra of Prepared Compounds}

$\left[\mathrm{R}_{\mathrm{F8}}\left(\mathrm{CH}_{2}\right)_{3}\right]\left(\mathrm{NC}-\mathrm{CH}_{2} \mathrm{CH}_{2}\right)_{3} \mathbf{P}^{+} \mathbf{I}^{-}$

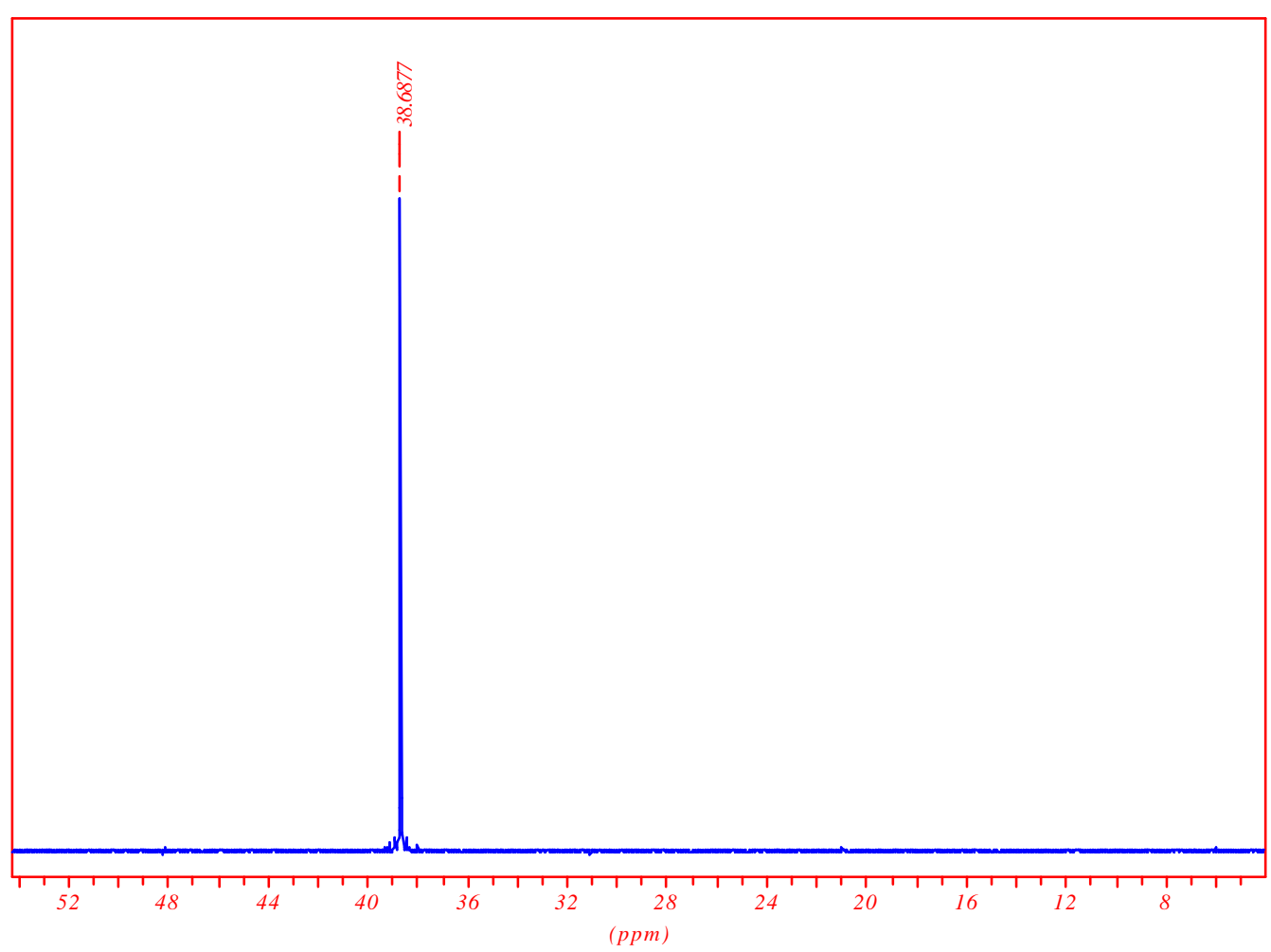




\section{$\left[\mathrm{R}_{\mathrm{F8}}\left(\mathrm{CH}_{2}\right)_{3}\right]\left(\mathrm{NC}-\mathrm{CH}_{2} \mathrm{CH}_{2}\right)_{2} \mathrm{P}$}

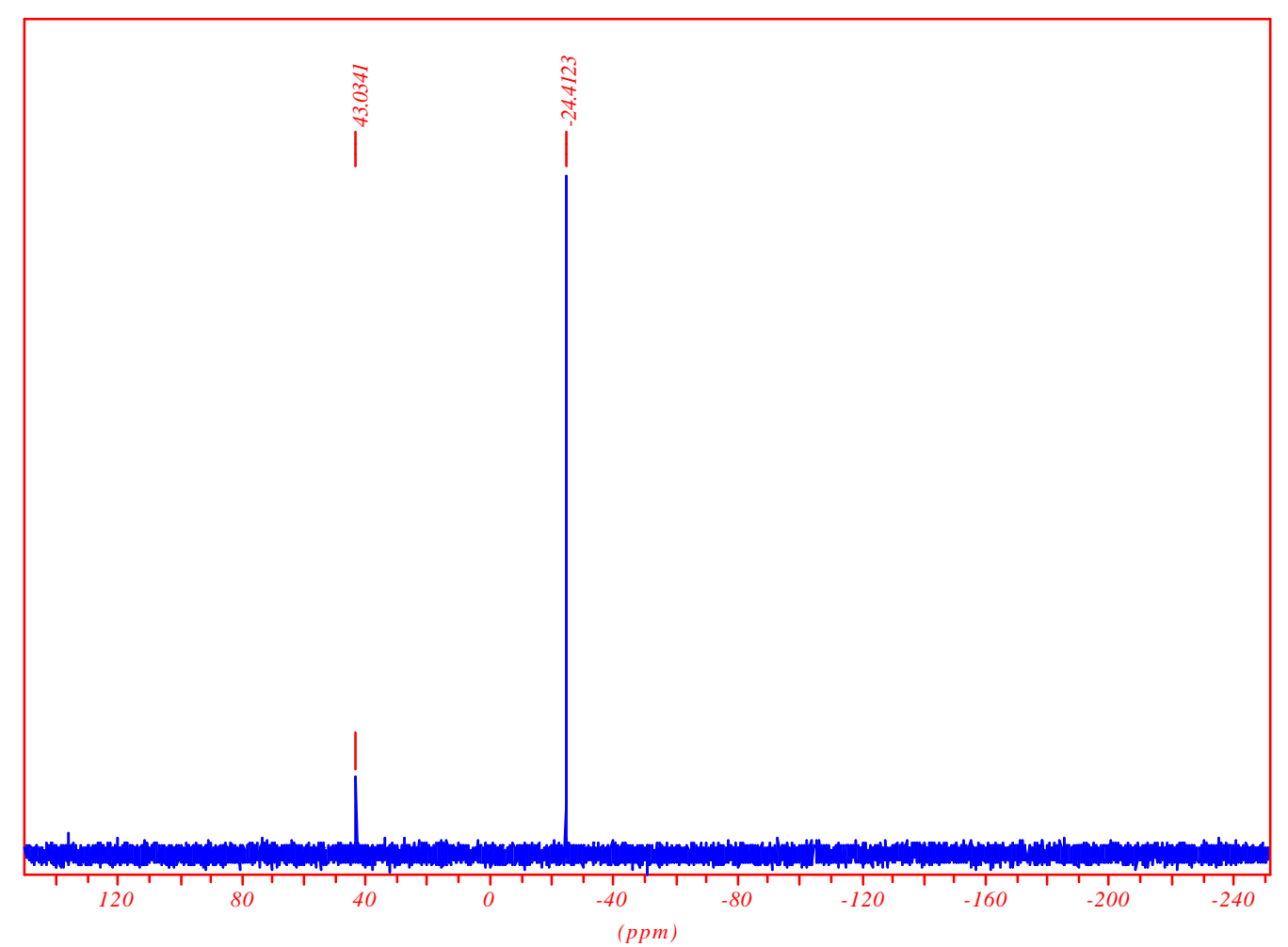


$\left[\mathbf{R}_{\mathrm{F}}\left(\mathrm{CH}_{2}\right)_{3}\right]_{2}\left(\mathrm{NC}-\mathrm{CH}_{2} \mathrm{CH}_{2}\right)_{2} \mathbf{P}^{+} \mathbf{I}^{-}$

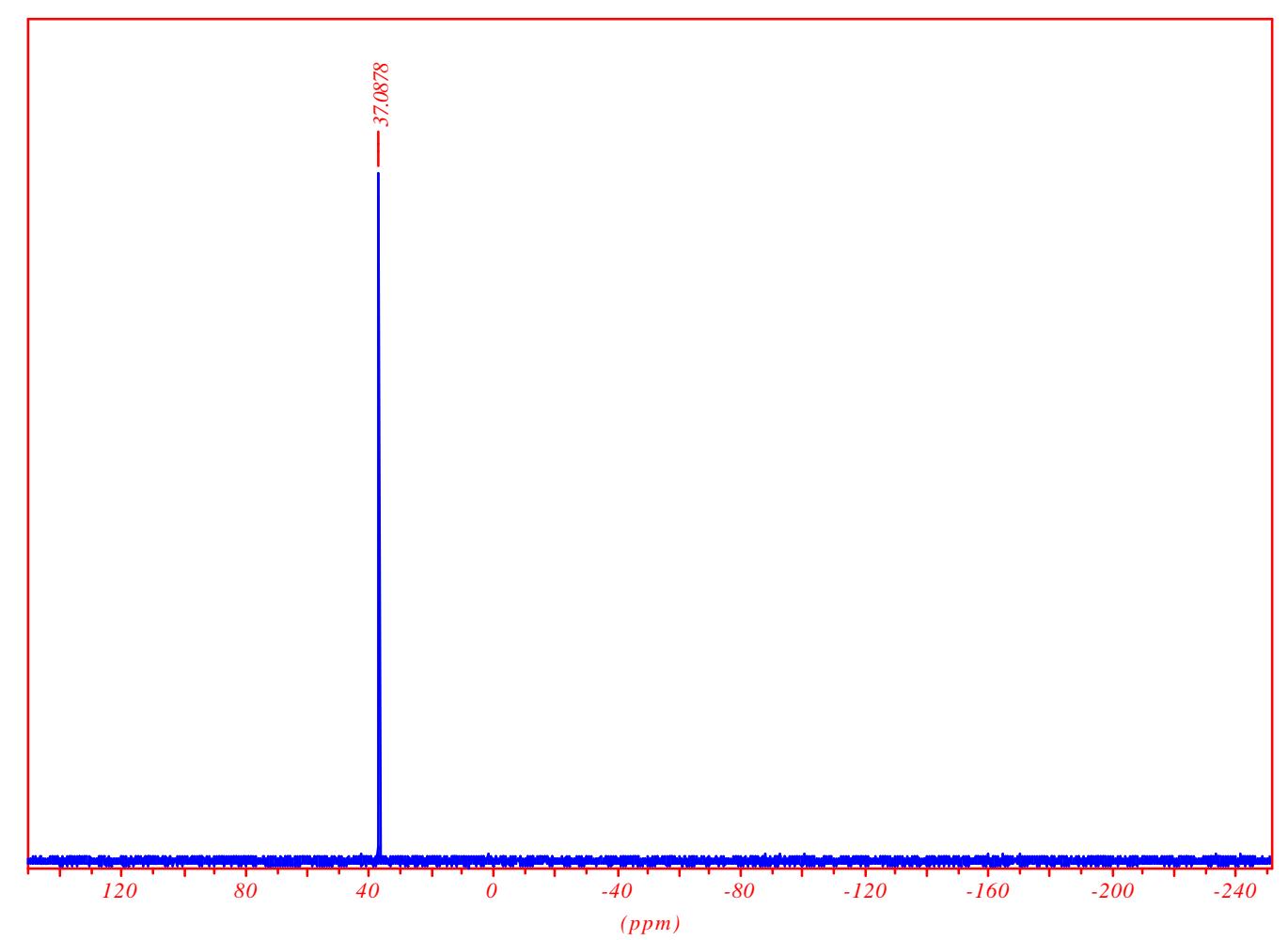


$\left[\mathrm{R}_{\mathrm{Fz}}\left(\mathrm{CH}_{2}\right)_{3}\right]_{2}\left(\mathrm{NC}-\mathrm{CH}_{2} \mathrm{CH}_{2}\right) \mathbf{P}$

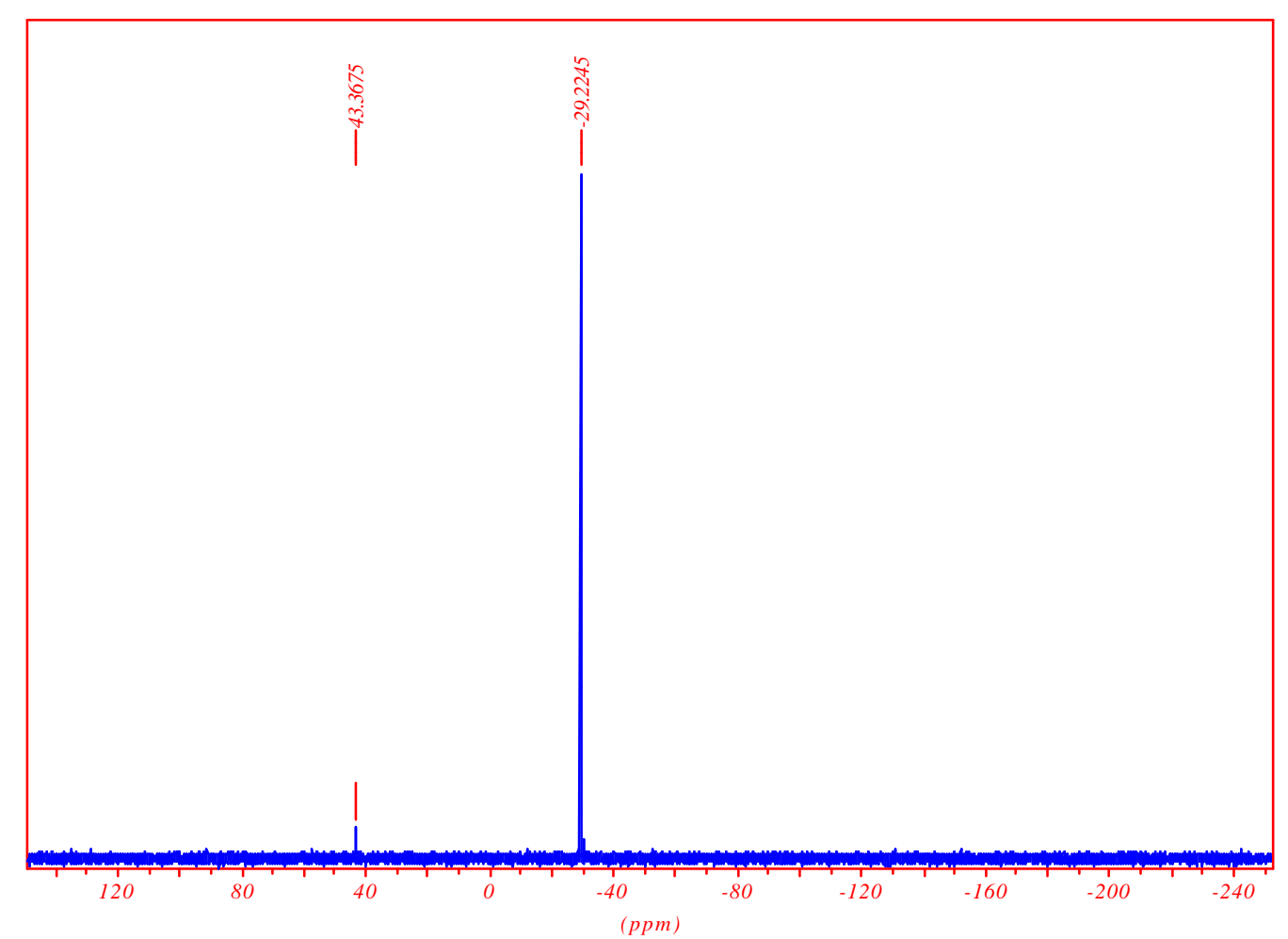


$\left[\mathbf{R}_{\mathrm{Fz}}\left(\mathrm{CH}_{2}\right)_{3}\right]_{3}\left(\mathrm{NC}-\mathrm{CH}_{2} \mathrm{CH}_{2}\right) \mathbf{P}^{+} \mathbf{I}^{-}$

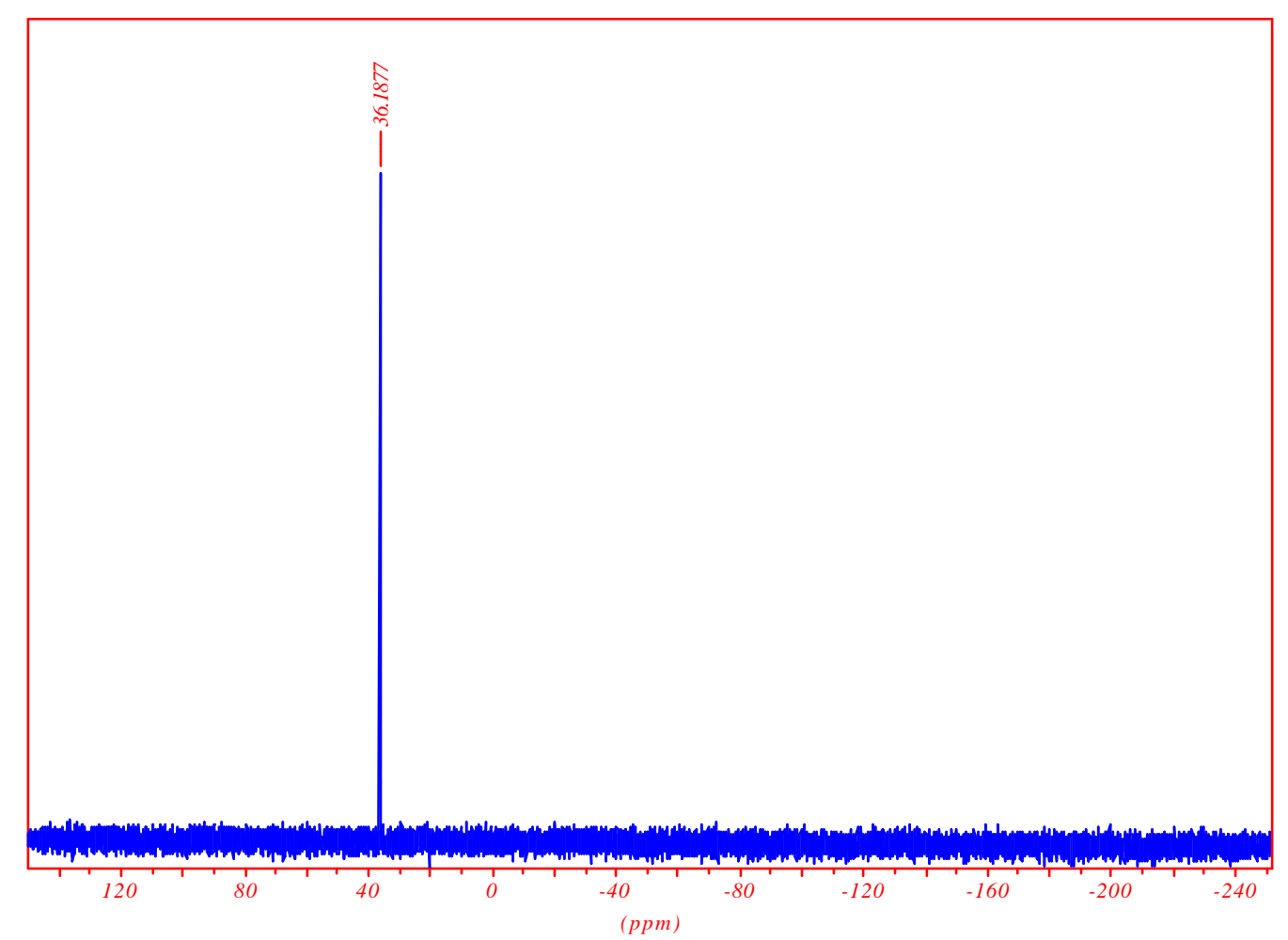


$\left[\mathbf{R}_{\mathbf{F 8}}\left(\mathbf{C H}_{2}\right)_{3}\right]_{3} \mathbf{P}$

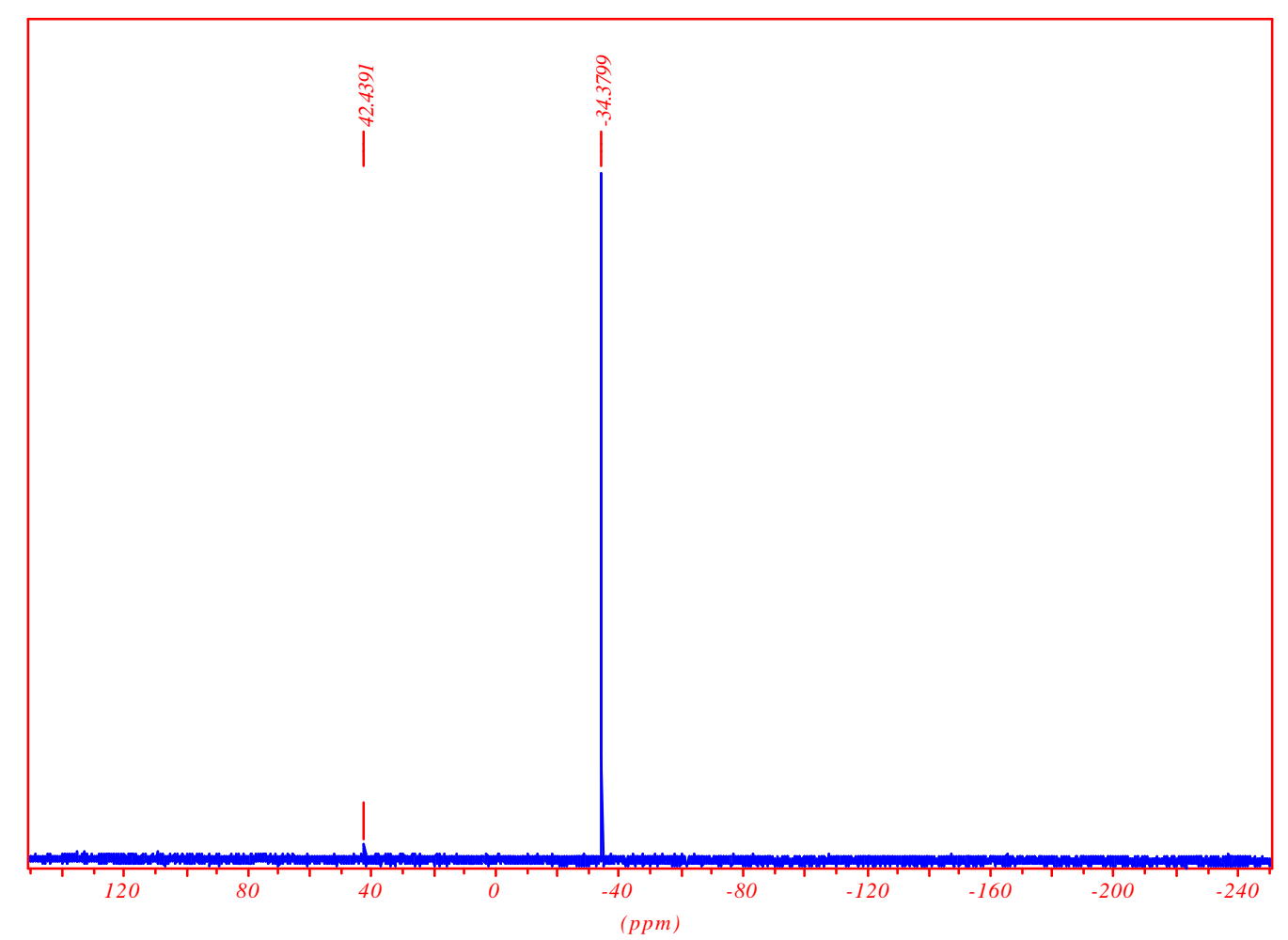


$\left[\mathrm{R}_{\mathrm{F6}}\left(\mathrm{CH}_{2}\right)_{3}\right]\left(\mathrm{NC}-\mathrm{CH}_{2} \mathrm{CH}_{2}\right)_{3} \mathbf{P}^{+} \mathbf{I}^{-}$

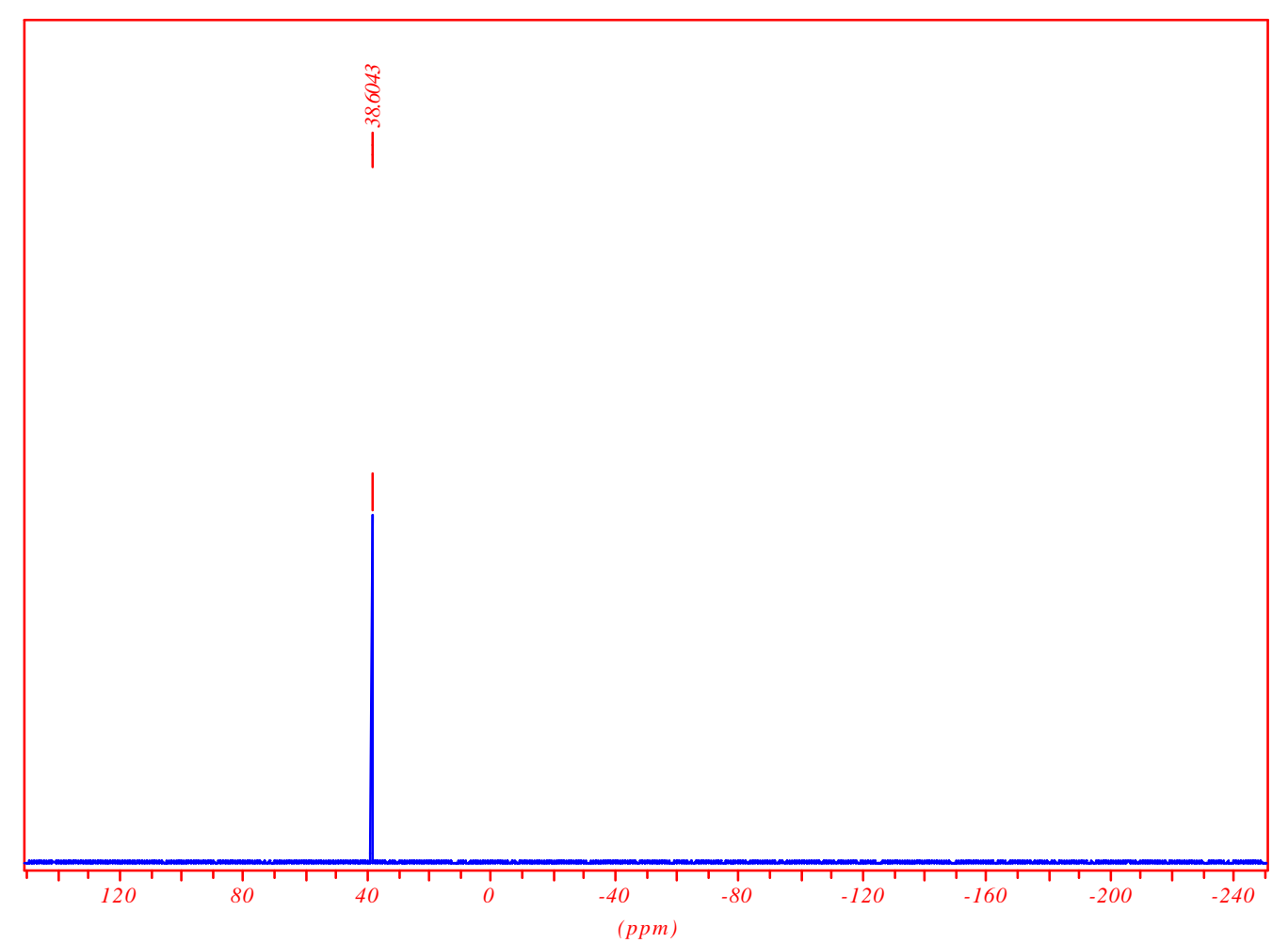


$\left[\mathbf{R}_{\mathrm{F} 6}\left(\mathrm{CH}_{2}\right)_{3}\right]\left(\mathrm{NC}-\mathrm{CH}_{2} \mathrm{CH}_{2}\right)_{2} \mathbf{P}$

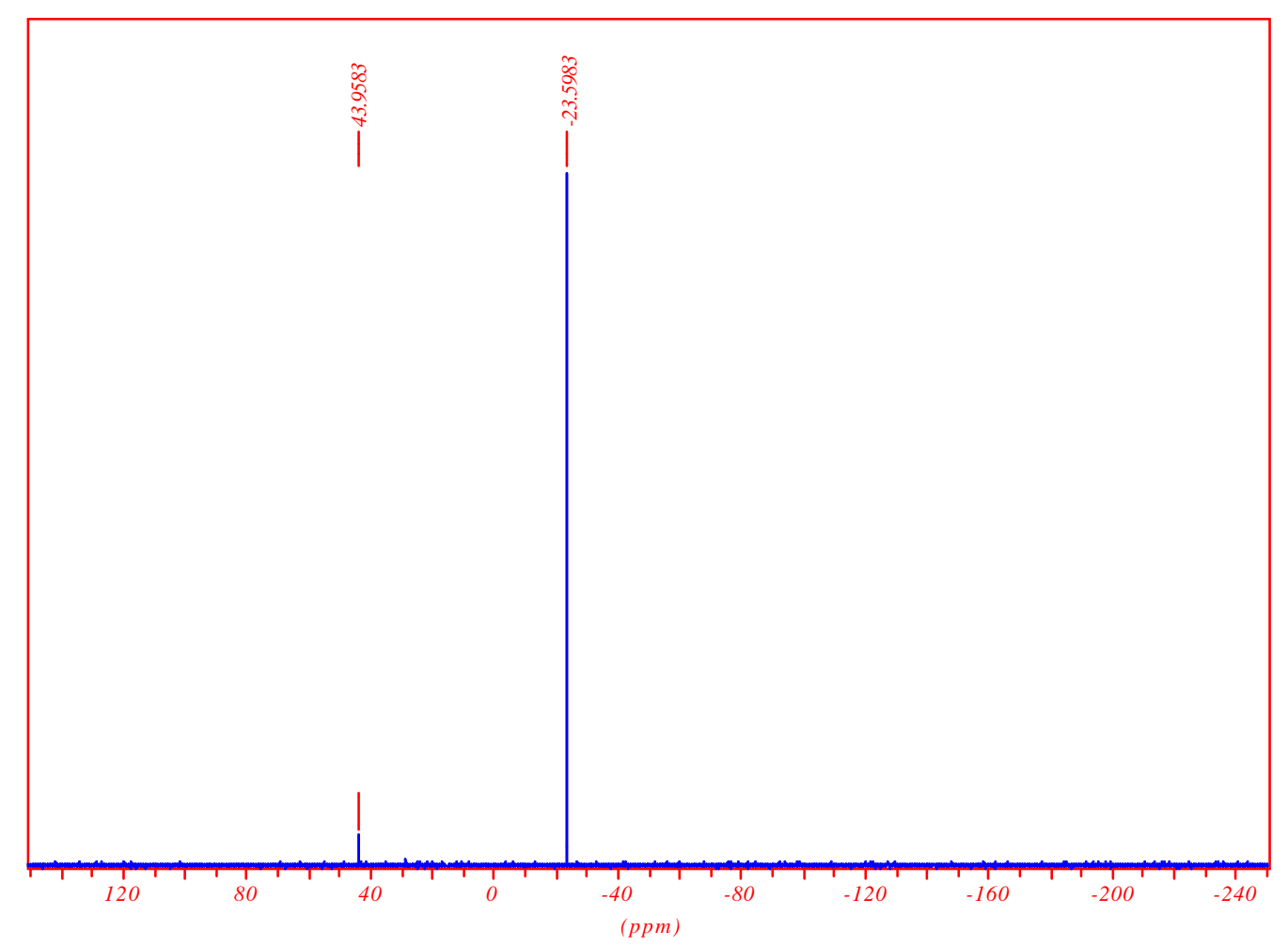


$\left[\mathbf{R}_{\mathrm{F} 6}\left(\mathrm{CH}_{2}\right)_{3}\right]_{2}\left(\mathrm{NC}-\mathrm{CH}_{2} \mathrm{CH}_{2}\right)_{2} \mathbf{P}^{+} \mathbf{I}^{-}$

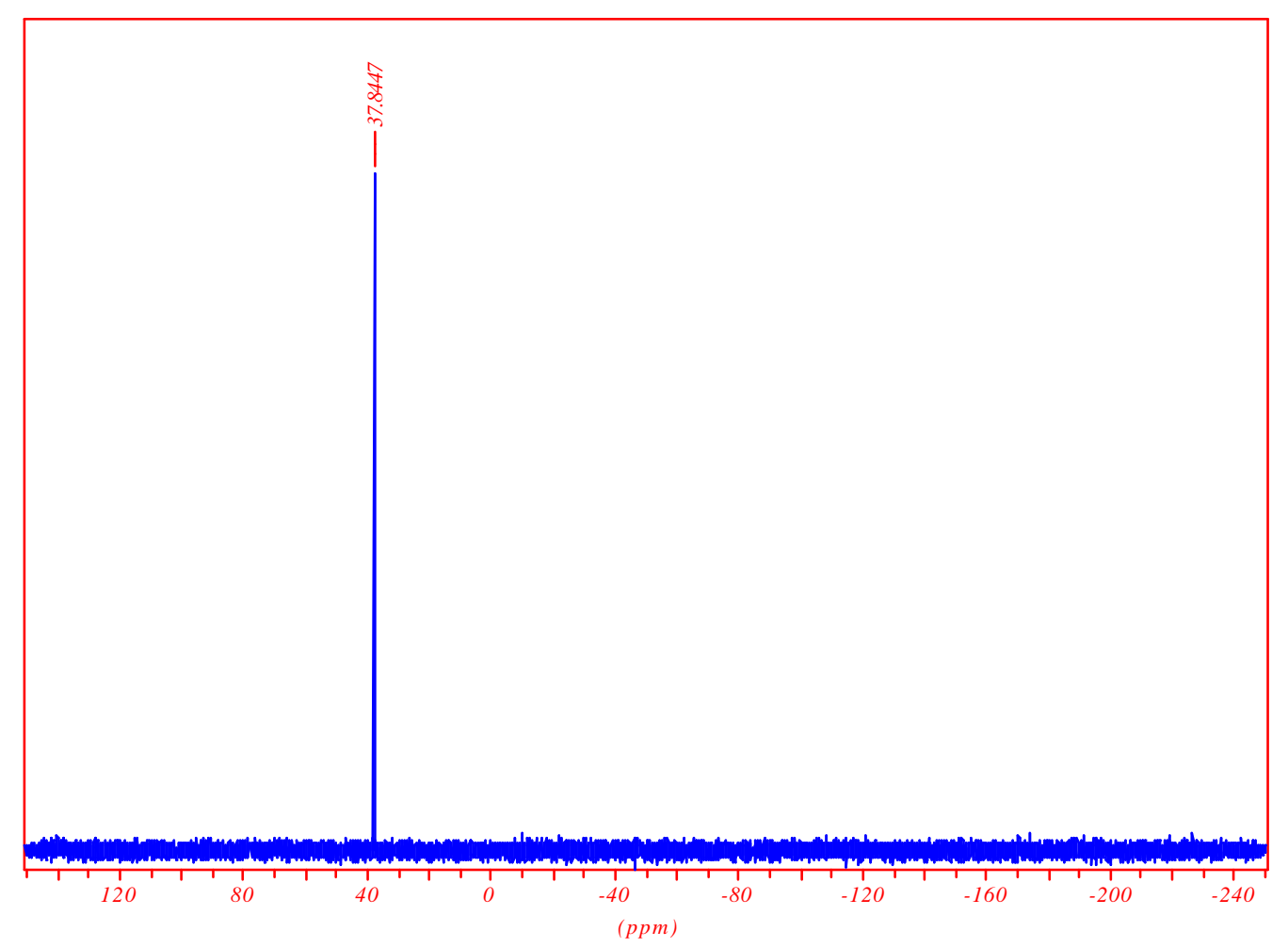


$\left[\mathbf{R}_{\mathrm{F} 6}\left(\mathrm{CH}_{2}\right)_{3}\right]_{2}\left(\mathrm{NC}-\mathrm{CH}_{2} \mathrm{CH}_{2}\right) \mathbf{P}$

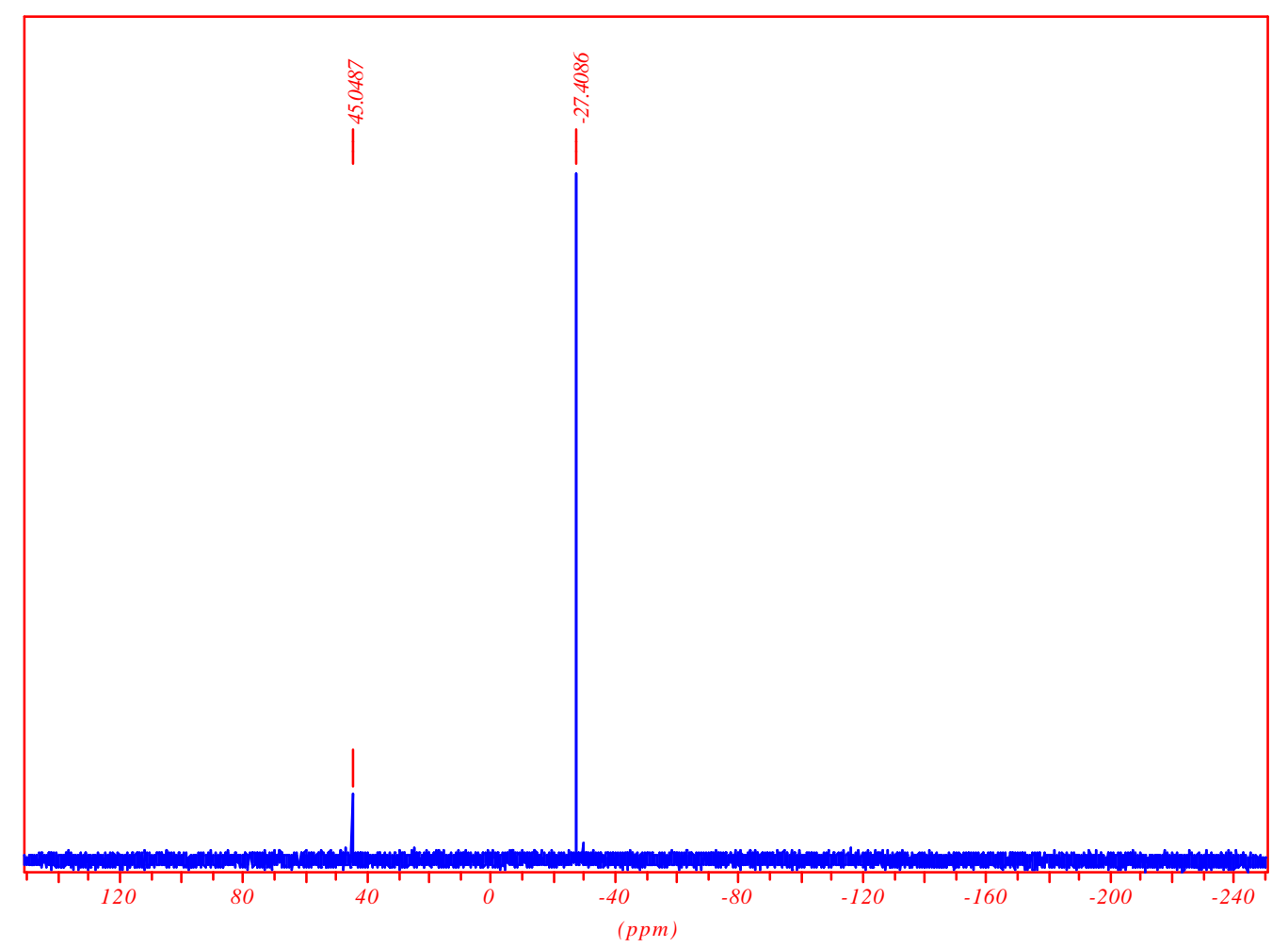


$\left[\mathbf{R}_{\mathrm{F} 6}\left(\mathrm{CH}_{2}\right)_{3}\right]_{3}\left(\mathrm{NC}-\mathrm{CH}_{2} \mathrm{CH}_{2}\right) \mathbf{P}^{+} \mathbf{I}^{-}$

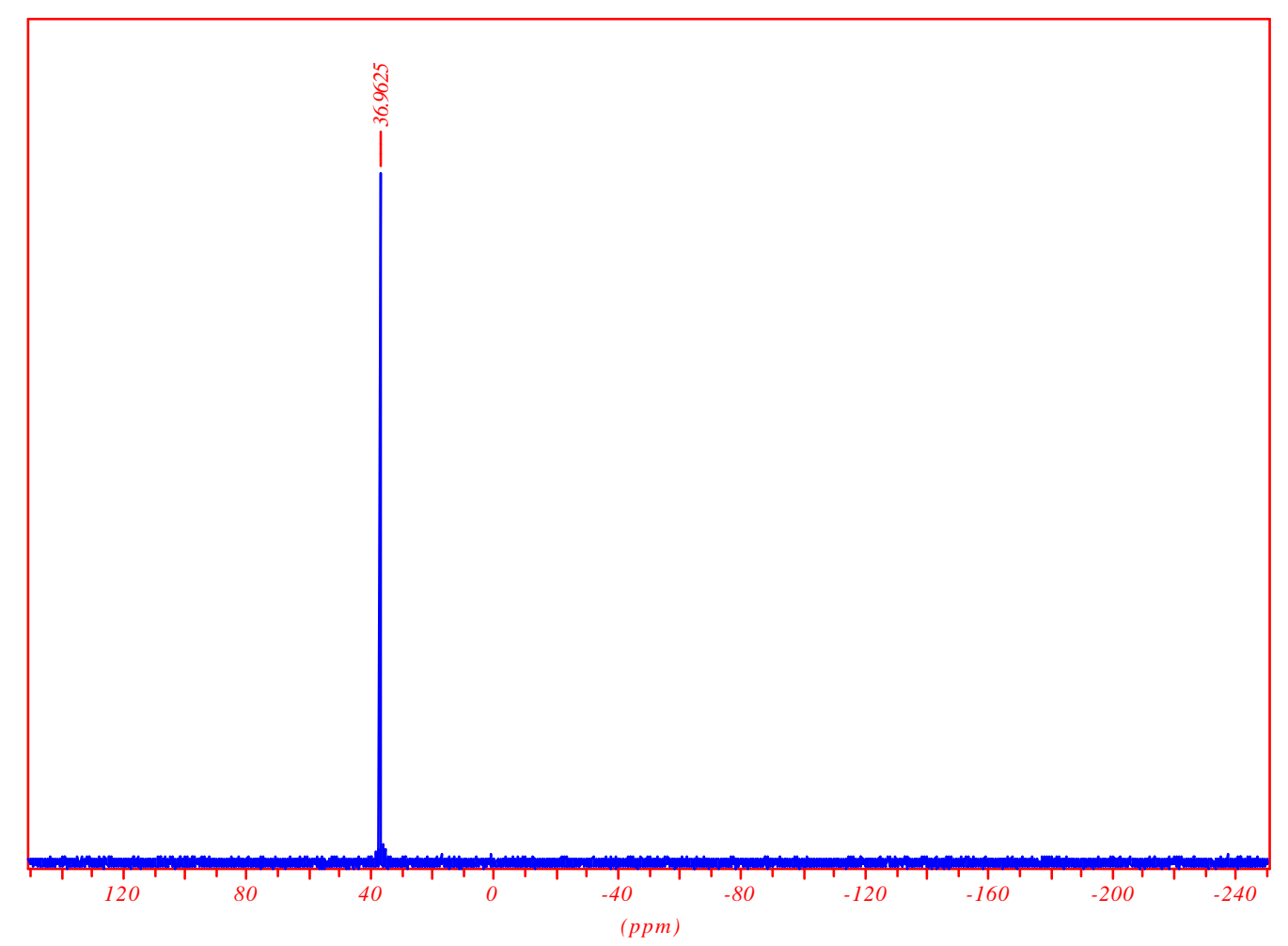


$\left[\mathbf{R}_{\mathrm{F} 6}\left(\mathrm{CH}_{2}\right)_{3}\right]_{3} \mathbf{P}$

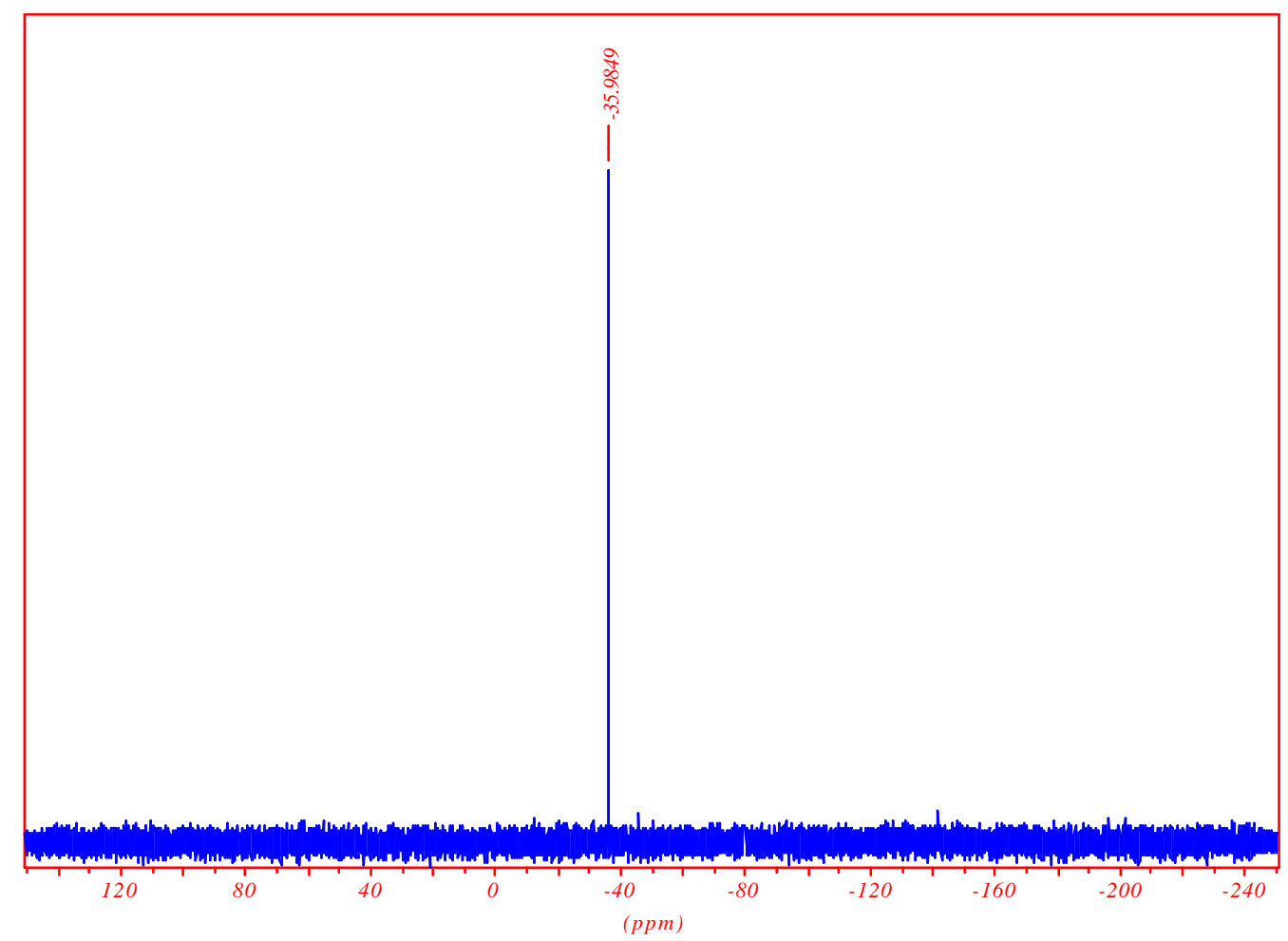


$\left[\mathbf{R}_{\mathrm{F} 4}\left(\mathbf{C H}_{2}\right)_{3}\right]\left(\mathrm{NC}-\mathrm{CH}_{2} \mathrm{CH}_{2}\right)_{3} \mathbf{P}^{+} \mathbf{I}$

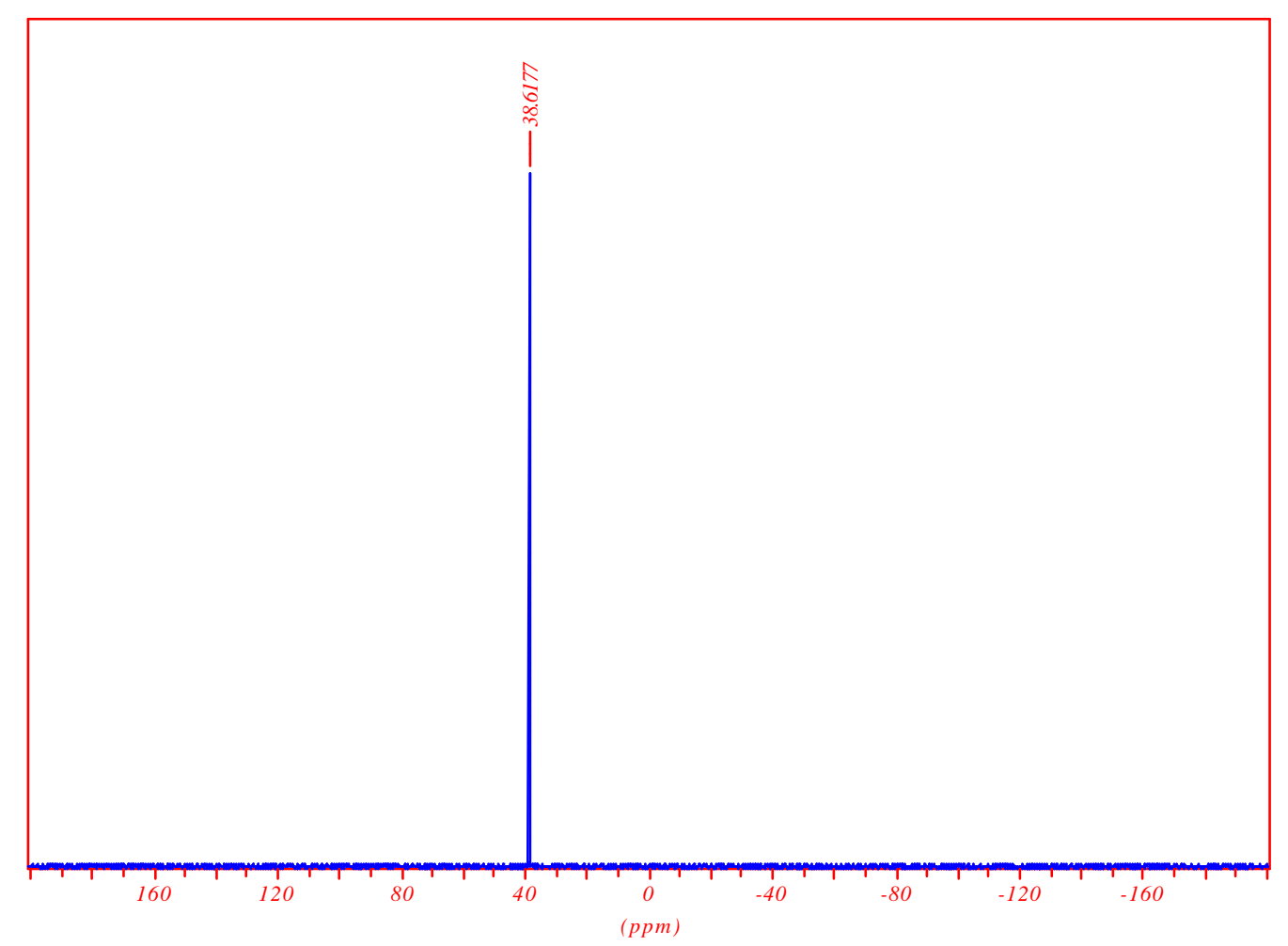




\section{$\left[\mathrm{R}_{\mathrm{F} 4}\left(\mathrm{CH}_{2}\right)_{3}\right]\left(\mathrm{NC}-\mathrm{CH}_{2} \mathrm{CH}_{2}\right)_{2} \mathbf{P}$}

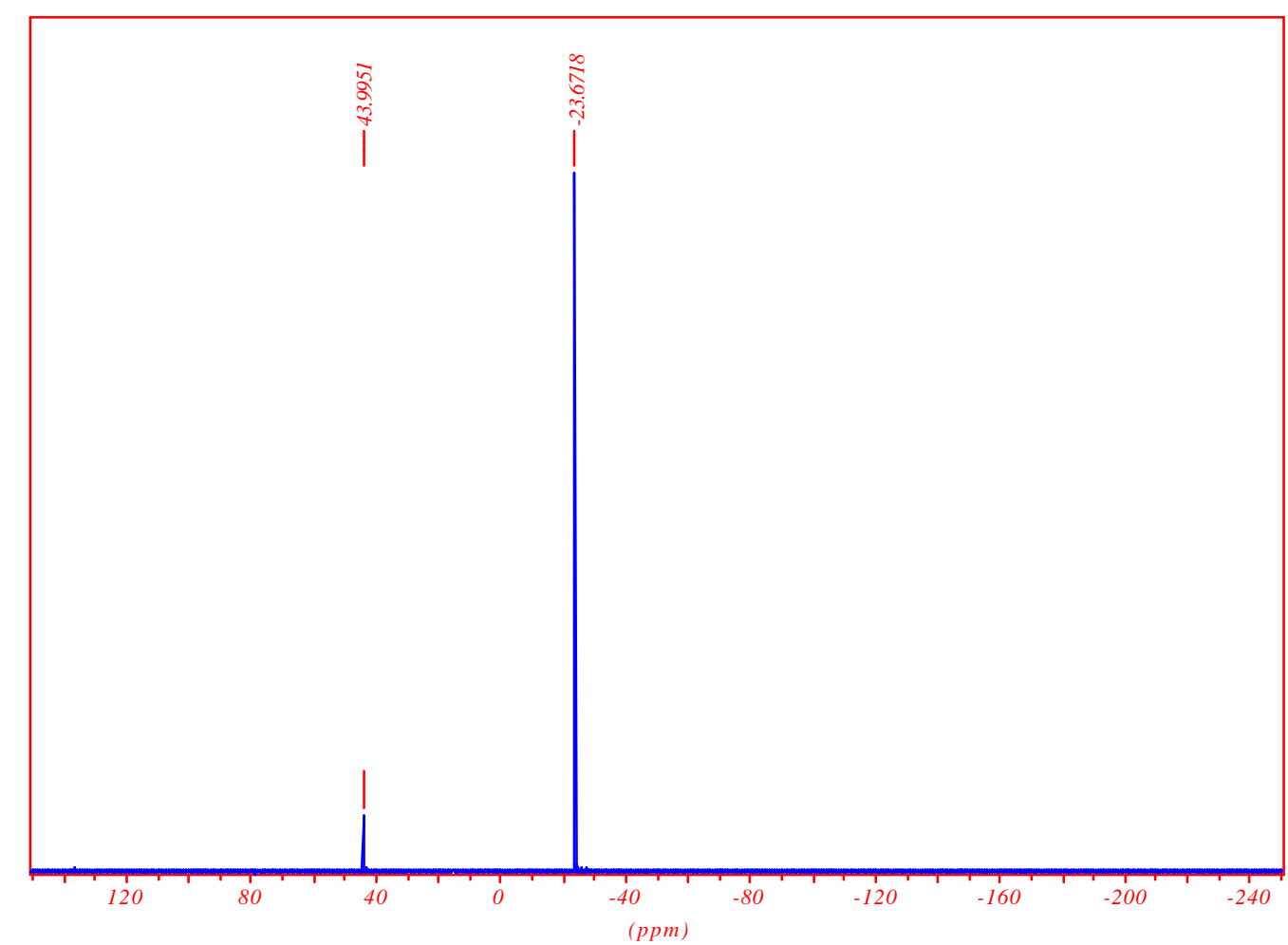


$\left[\mathbf{R}_{\mathrm{F} 4}\left(\mathrm{CH}_{2}\right)_{3}\right]_{2}\left(\mathrm{NC}-\mathrm{CH}_{2} \mathrm{CH}_{2}\right)_{2} \mathbf{P}^{+} \mathbf{I}^{-}$

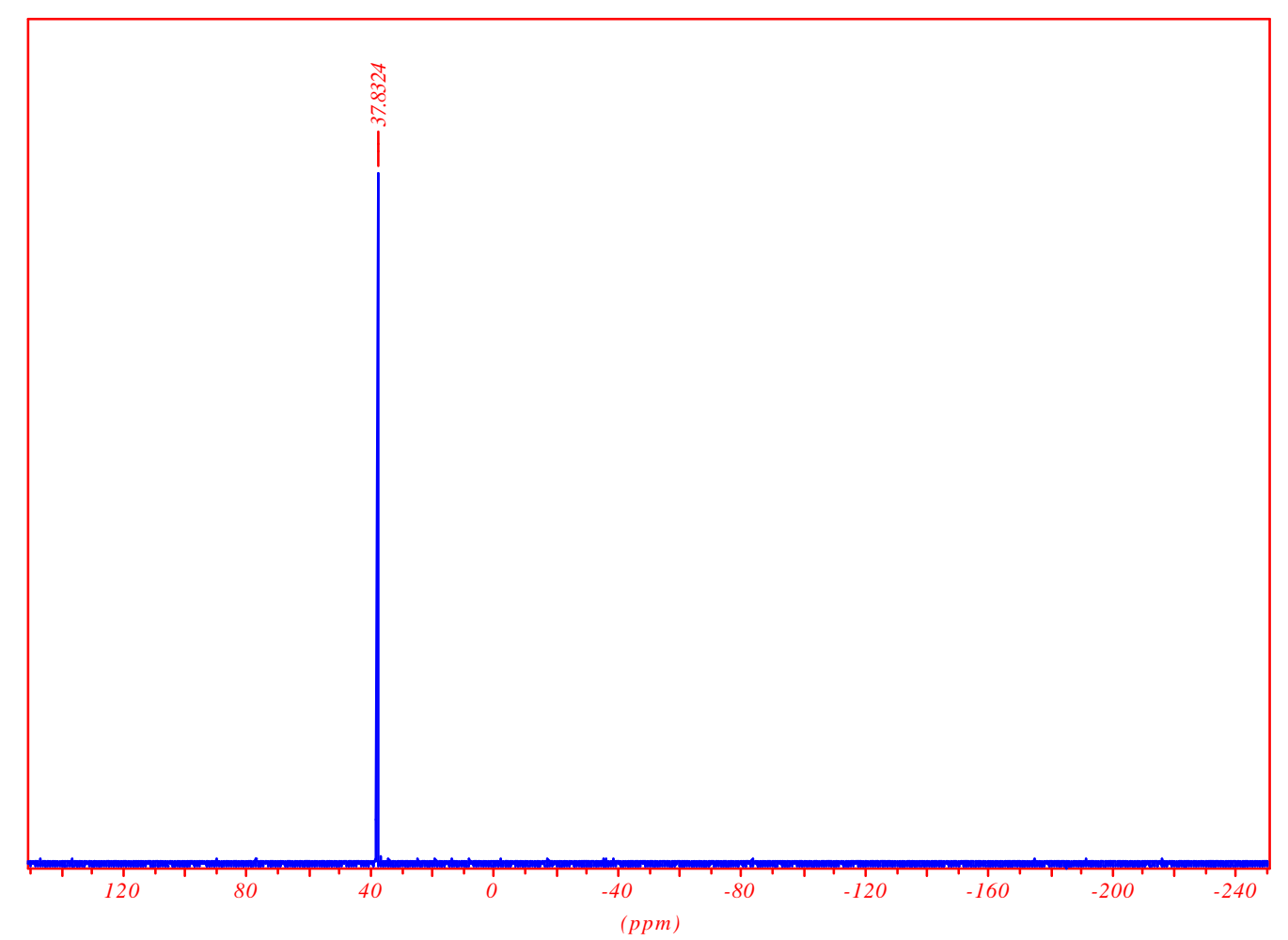


$\left[\mathbf{R}_{\mathrm{F} 4}\left(\mathrm{CH}_{2}\right)_{3}\right]_{2}\left(\mathrm{NC}-\mathrm{CH}_{2} \mathrm{CH}_{2}\right) \mathbf{P}$

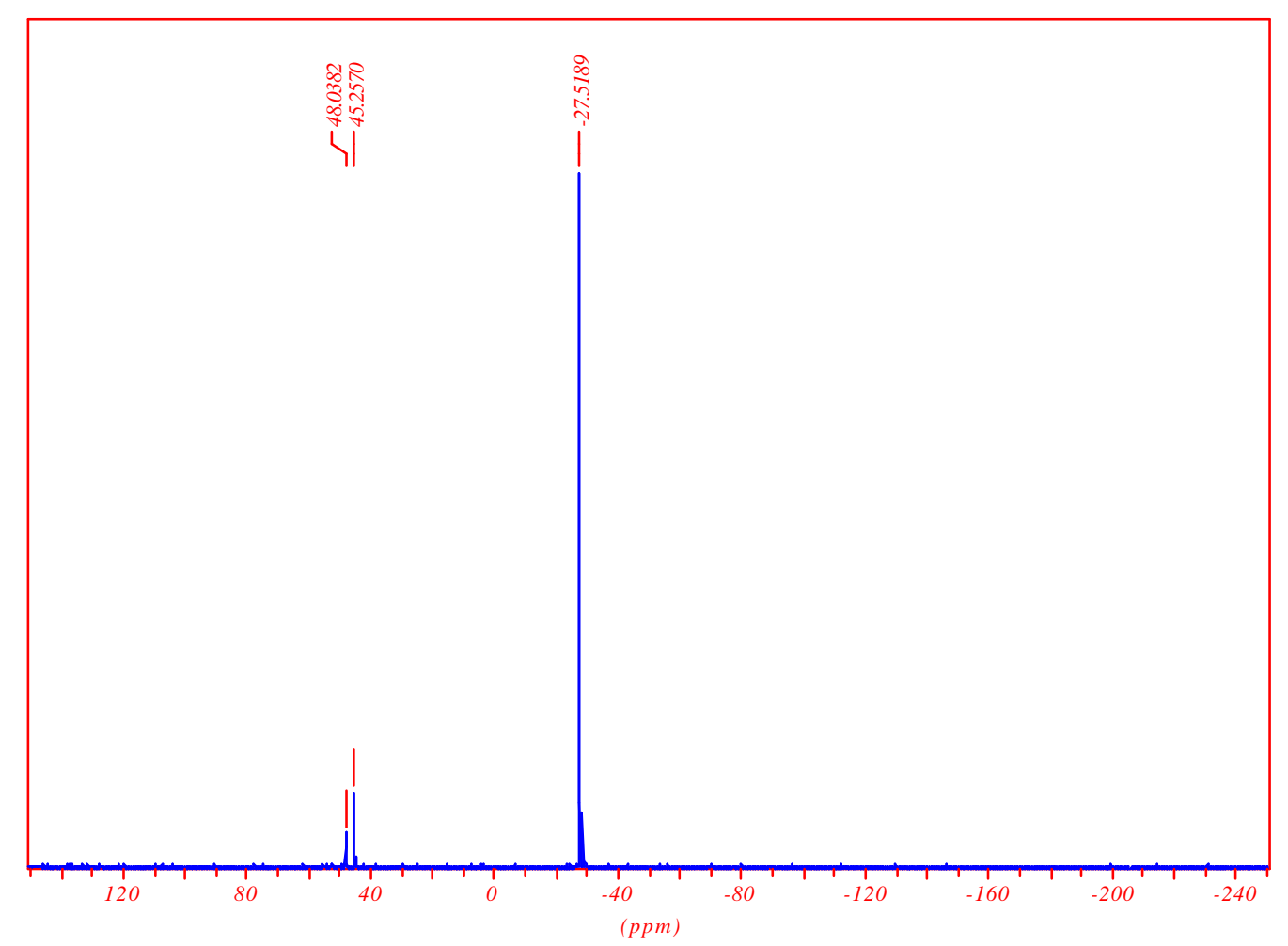


$\left[\mathrm{R}_{\mathrm{F} 4}\left(\mathrm{CH}_{2}\right)_{3}\right]_{3}\left(\mathrm{NC}-\mathrm{CH}_{2} \mathrm{CH}_{2}\right) \mathbf{P}^{+} \mathbf{I}^{-}$

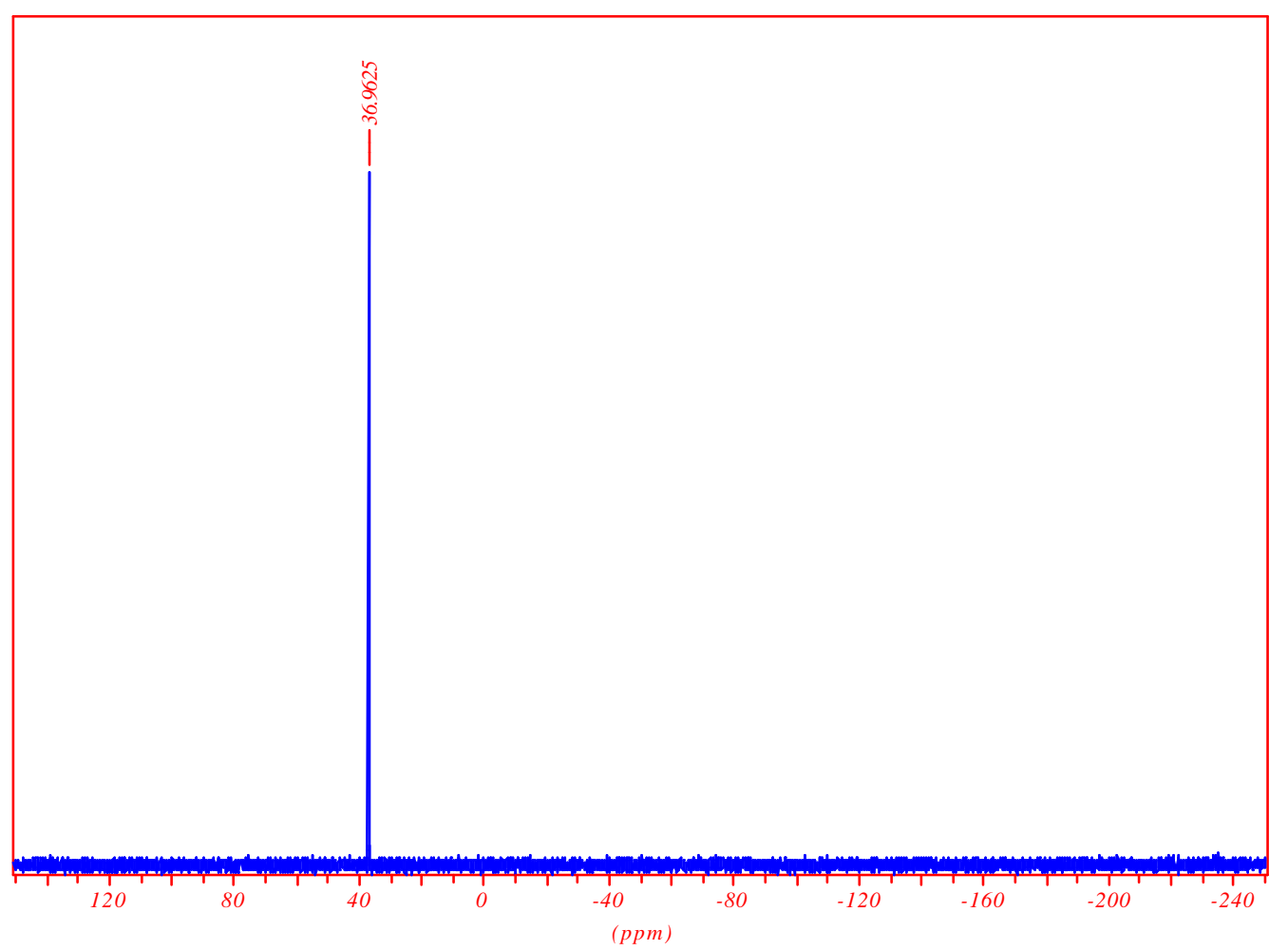


$\left[\mathbf{R}_{\mathrm{F4}}\left(\mathrm{CH}_{2}\right)_{3}\right]_{3} \mathbf{P}$

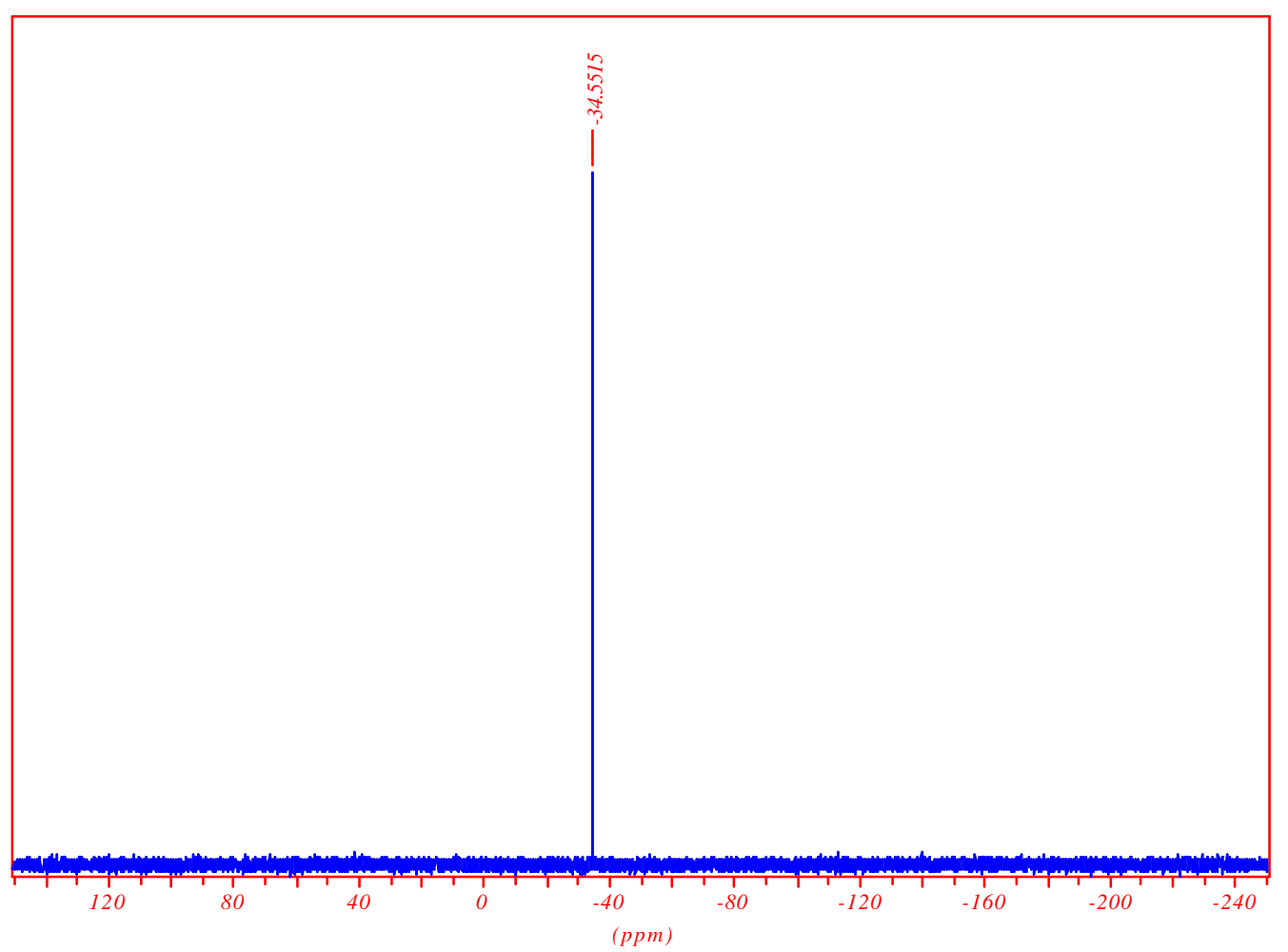


$\left[\mathrm{R}_{\mathrm{F8}}\left(\mathrm{CH}_{2}\right)_{4}\right]\left(\mathrm{NC}-\mathrm{CH}_{2} \mathrm{CH}_{2}\right)_{3} \mathbf{P}^{+} \mathbf{I}^{-}$

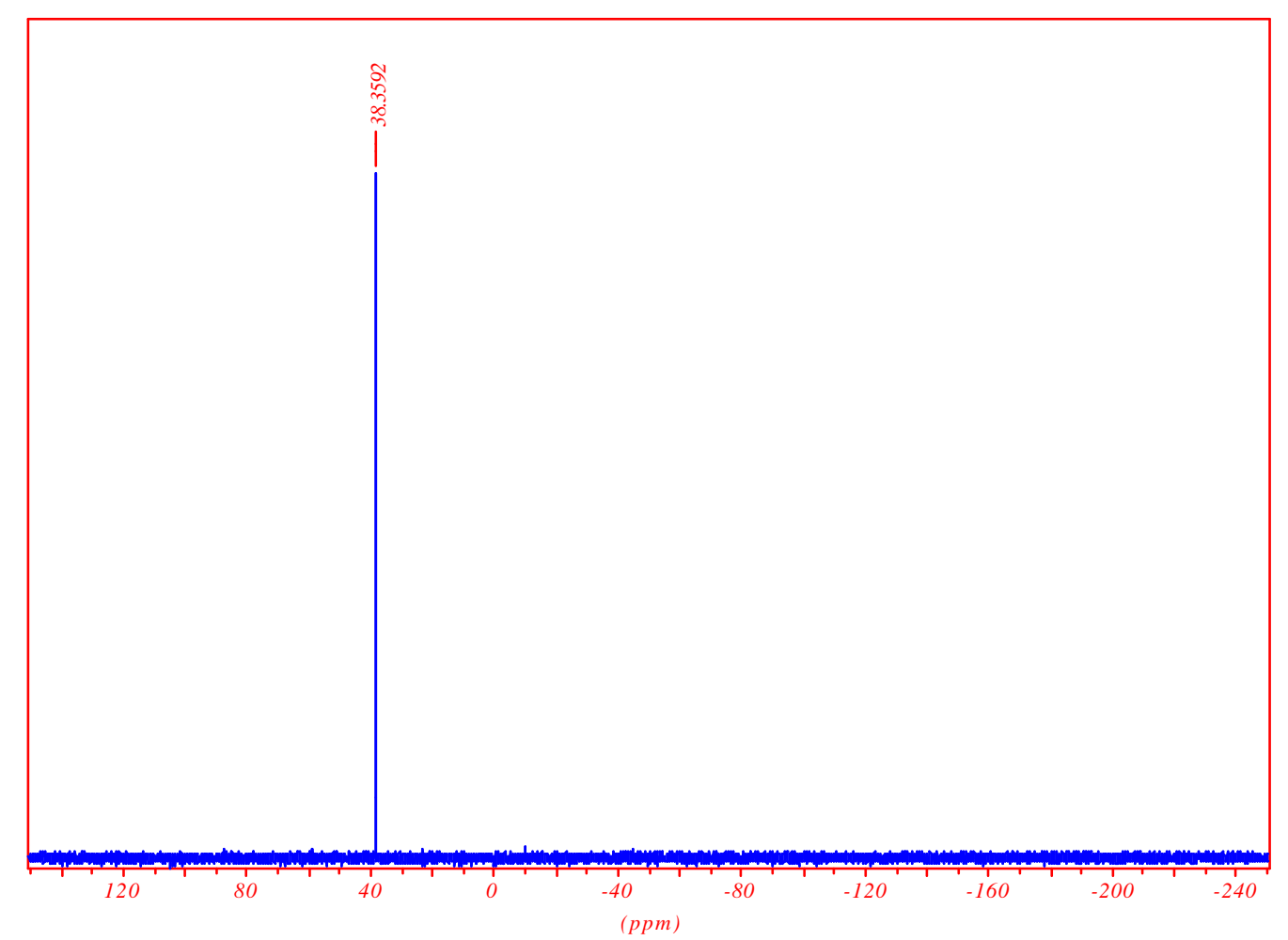


$\left[\mathrm{R}_{\mathrm{F8}}\left(\mathrm{CH}_{2}\right)_{4}\right]\left(\mathrm{NC}-\mathrm{CH}_{2} \mathrm{CH}_{2}\right)_{2} \mathrm{P}$

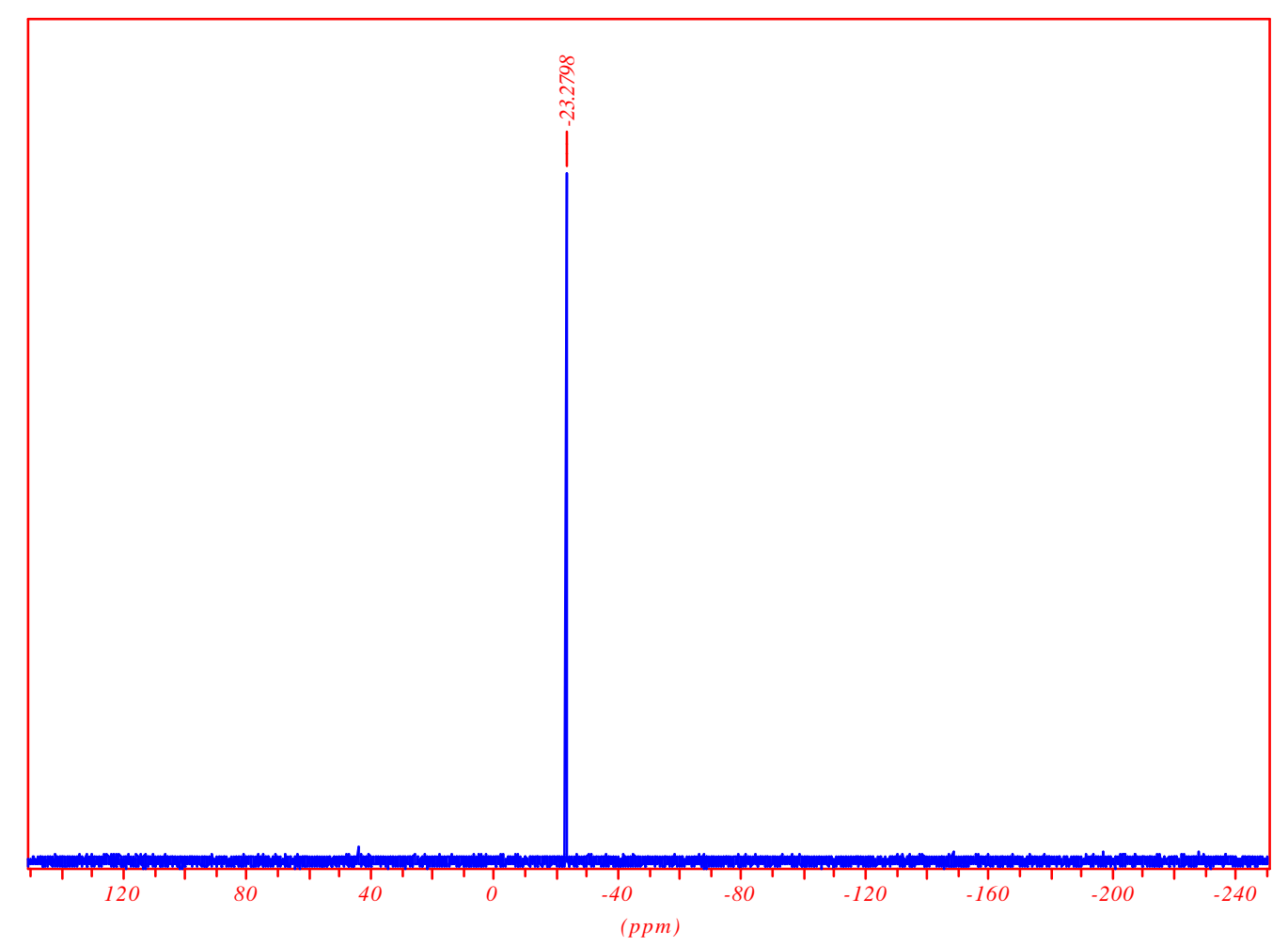


$\left[\mathbf{R}_{\mathrm{F}}\left(\mathrm{CH}_{2}\right)_{4}\right]_{2}\left(\mathrm{NC}-\mathrm{CH}_{2} \mathrm{CH}_{2}\right)_{2} \mathbf{P}^{+} \mathbf{I}^{-}$

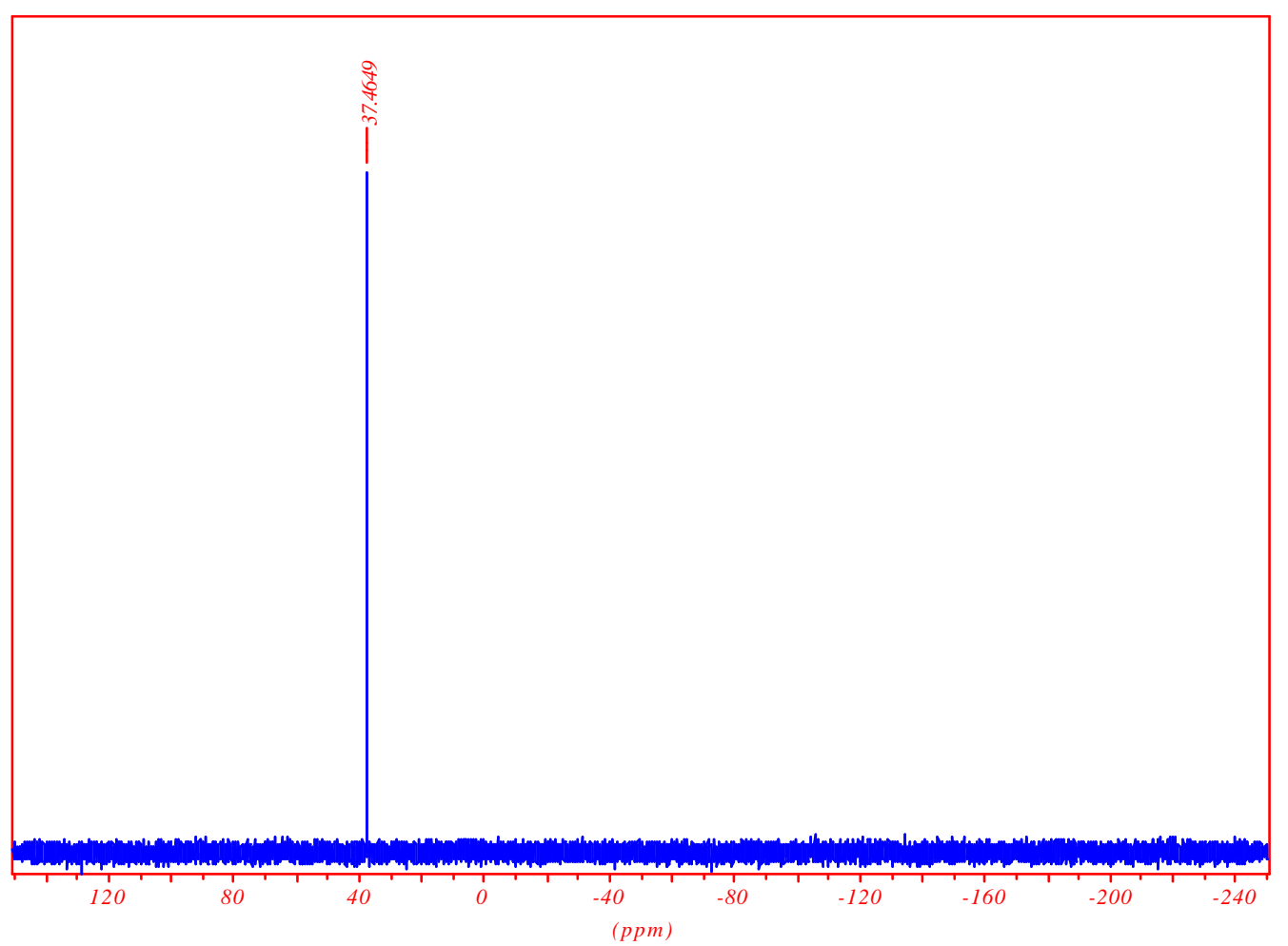


$\left[\mathrm{R}_{\mathrm{F8}}\left(\mathrm{CH}_{2}\right)_{4}\right]_{2}\left(\mathrm{NC}-\mathrm{CH}_{2} \mathrm{CH}_{2}\right) \mathrm{P}$

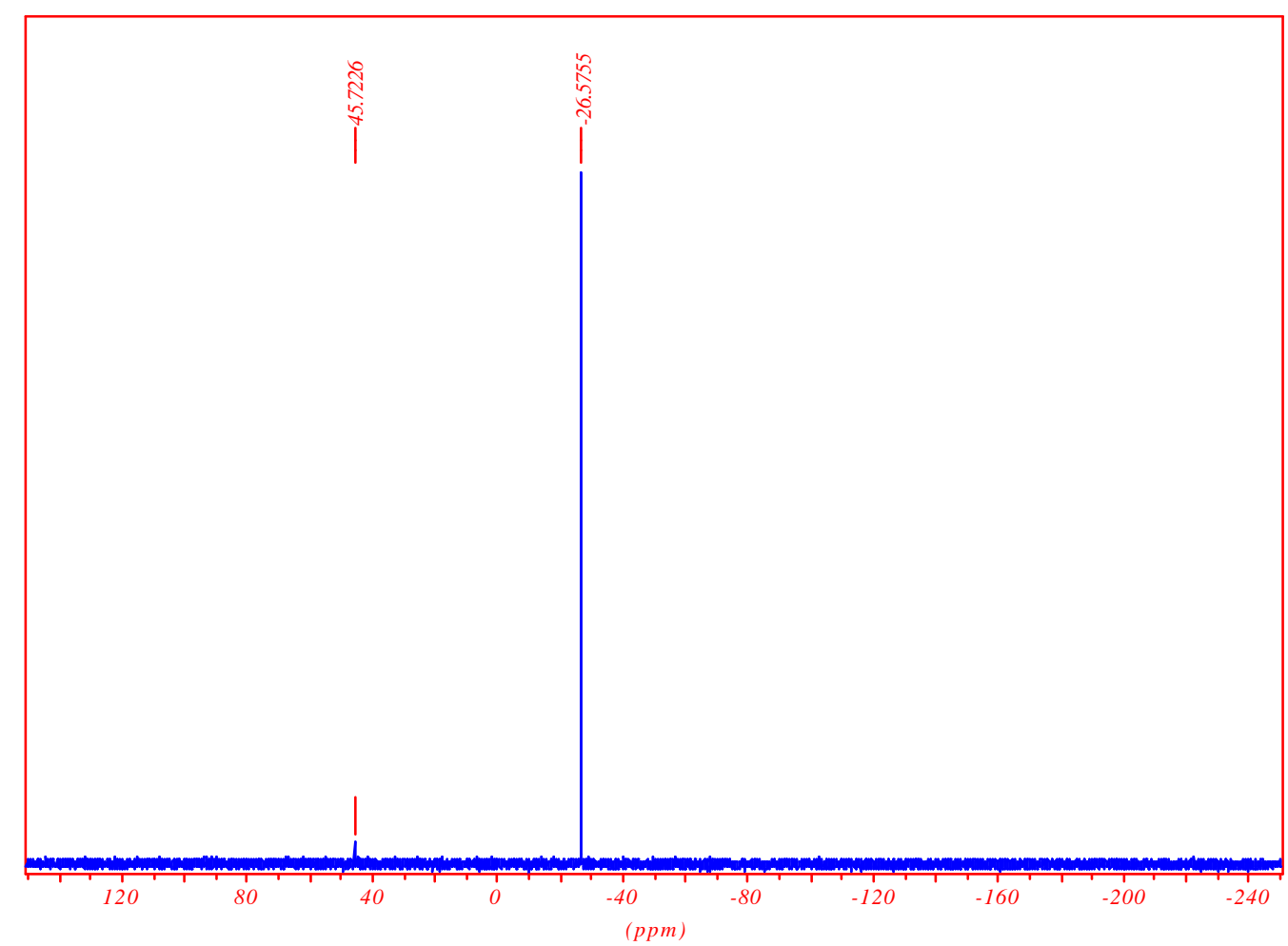


$\left[\mathrm{R}_{\mathrm{F8}}\left(\mathrm{CH}_{2}\right)_{4}\right]_{3}\left(\mathrm{NC}-\mathrm{CH}_{2} \mathrm{CH}_{2}\right) \mathbf{P}^{+} \mathbf{I}^{-}$

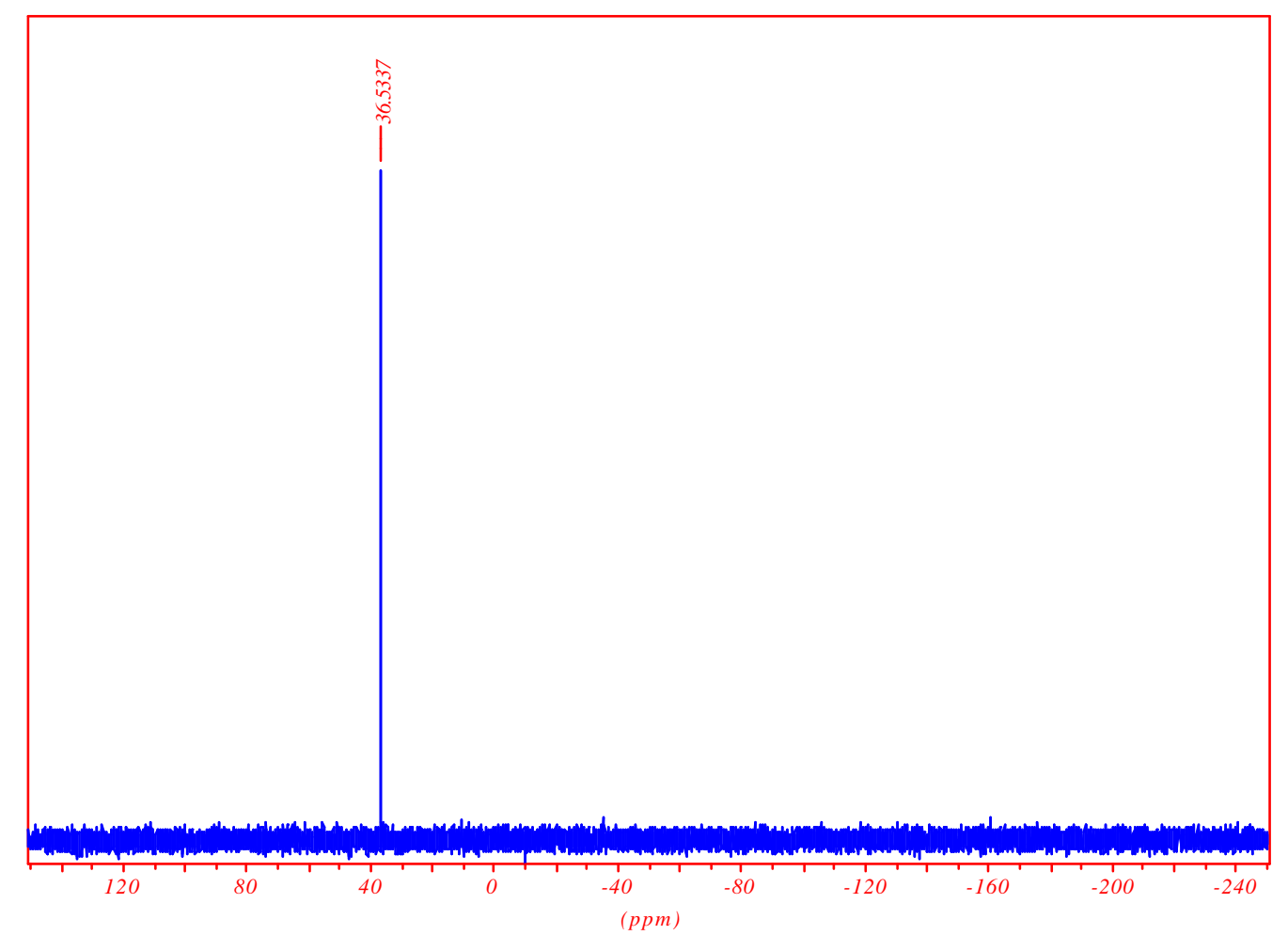


$\left[\mathbf{R}_{\mathrm{F8}}\left(\mathrm{CH}_{2}\right)_{4}\right]_{3} \mathbf{P}$

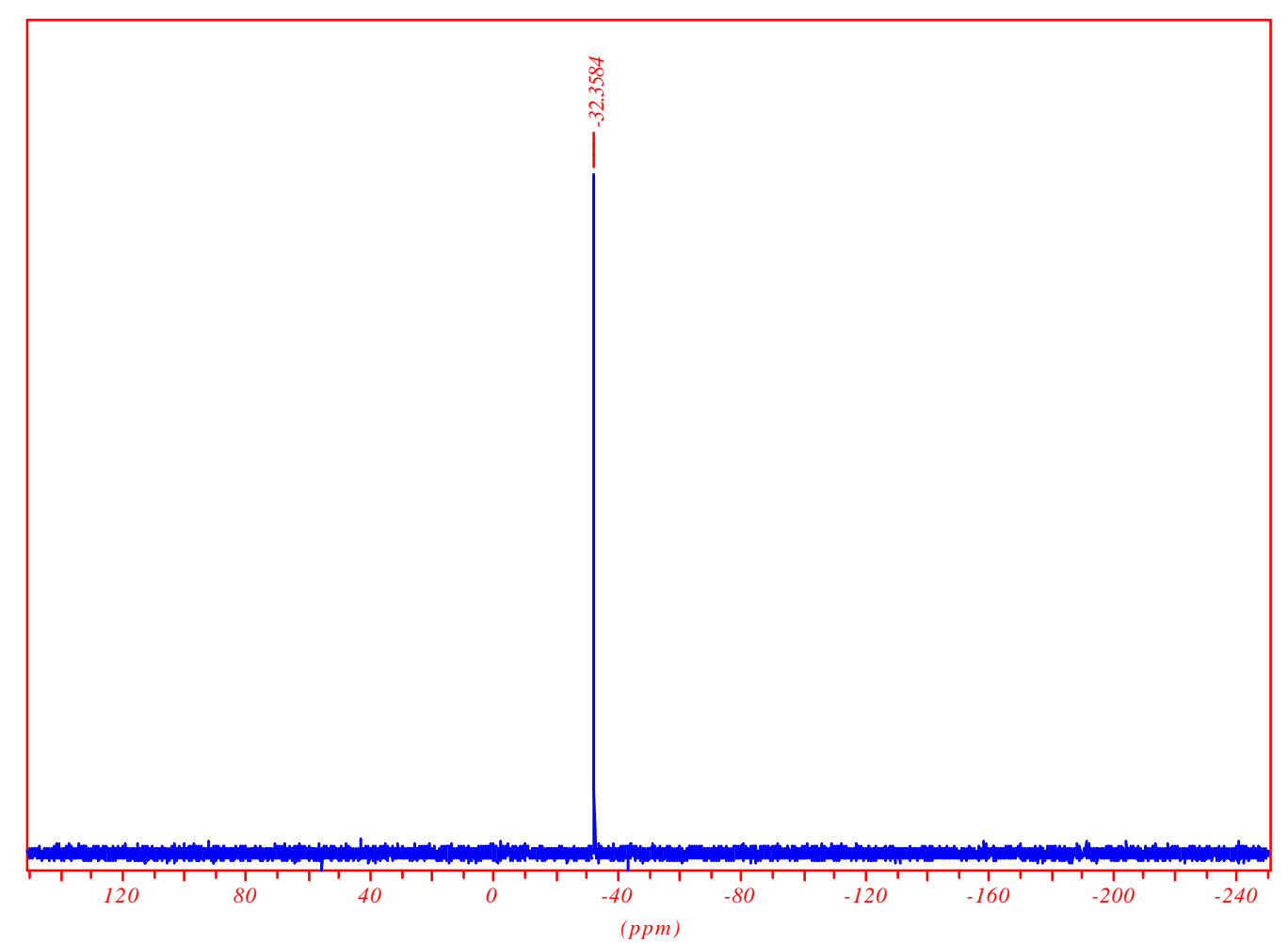


$\left[\mathrm{R}_{\mathrm{F8}}\left(\mathrm{CH}_{2}\right)_{3}\right]\left(\mathrm{NC}-\mathrm{CH}_{2} \mathrm{CH}_{2}\right)_{3} \mathrm{P}^{+} \mathbf{I}^{-}$

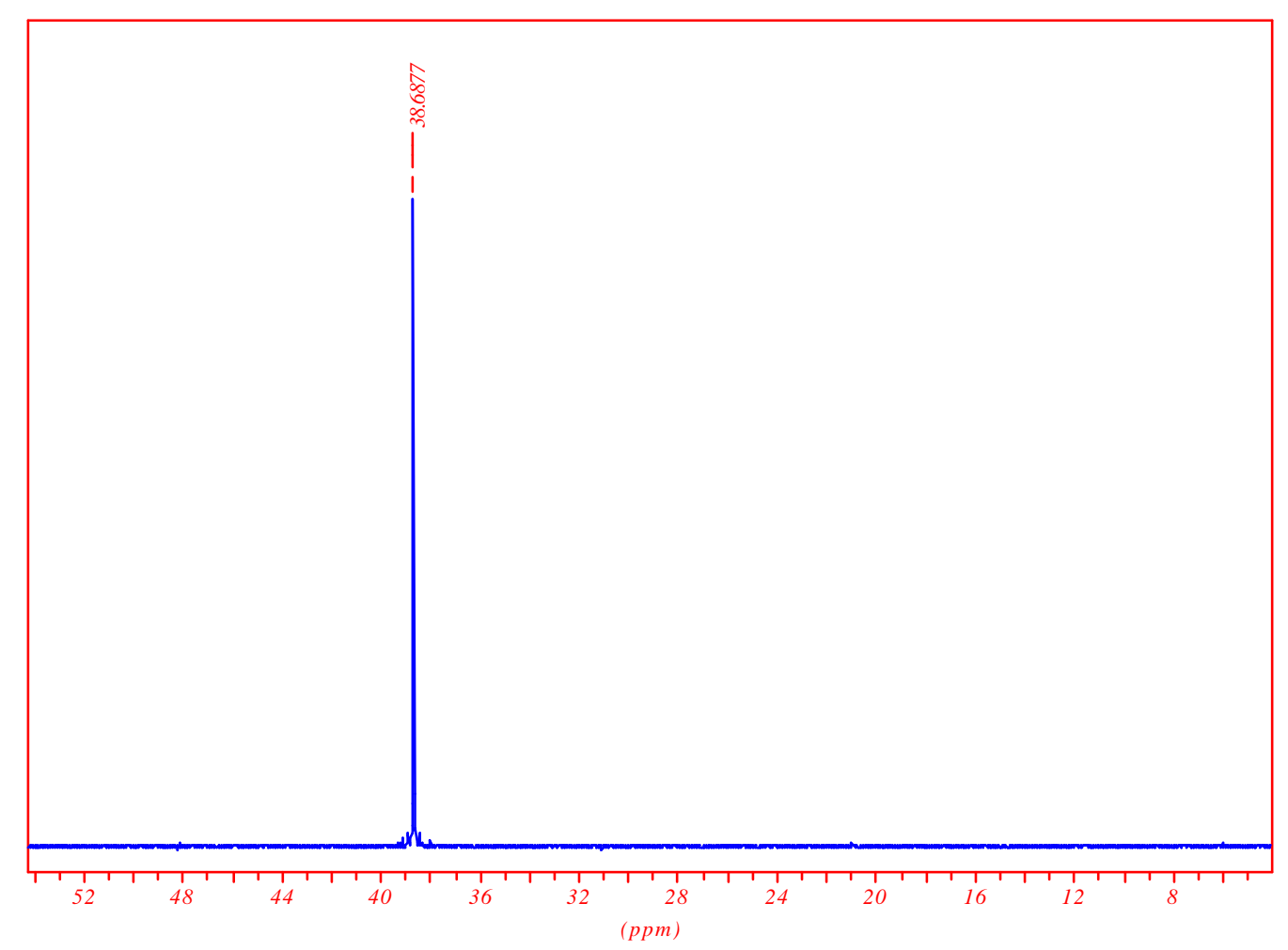




\section{$\left[\mathrm{R}_{\mathrm{F8}}\left(\mathrm{CH}_{2}\right)_{3}\right]\left(\mathrm{NC}-\mathrm{CH}_{2} \mathrm{CH}_{2}\right)_{2} \mathrm{P}$}

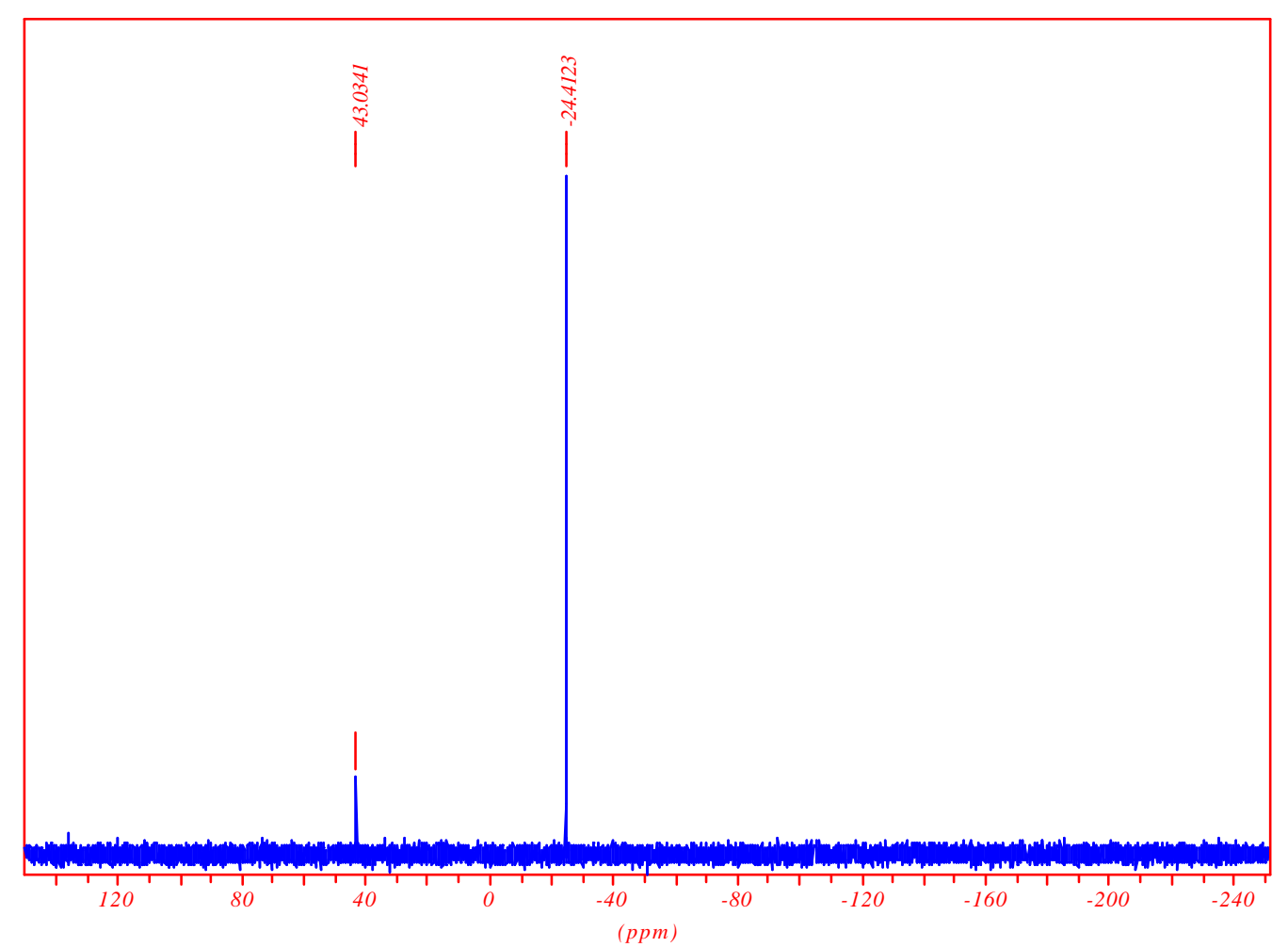


$\left[\mathbf{R}_{\mathrm{FP}}\left(\mathrm{CH}_{2}\right)_{3}\right]\left[\mathbf{R}_{\mathrm{F8}}\left(\mathrm{CH}_{2}\right)_{4}\right]\left(\mathrm{NC}-\mathrm{CH}_{2} \mathrm{CH}_{2}\right)_{2} \mathbf{P}^{+} \mathbf{I}^{-}$

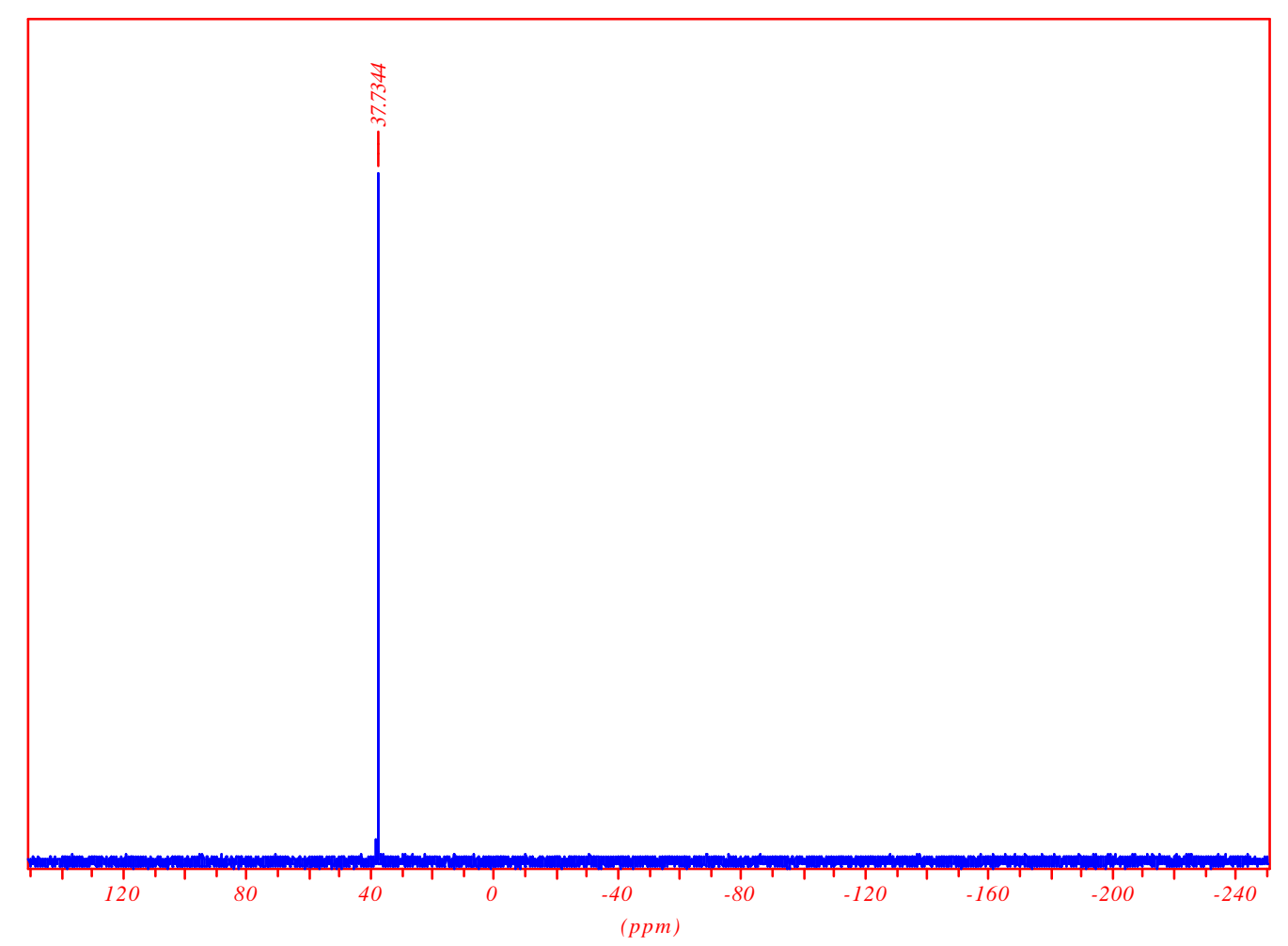


$\left[\mathbf{R}_{\mathrm{F8}}\left(\mathrm{CH}_{2}\right)_{3}\right]\left[\mathbf{R}_{\mathrm{F8}}\left(\mathrm{CH}_{2}\right)_{4}\right]\left(\mathrm{NC}-\mathrm{CH}_{2} \mathrm{CH}_{2}\right) \mathbf{P}$

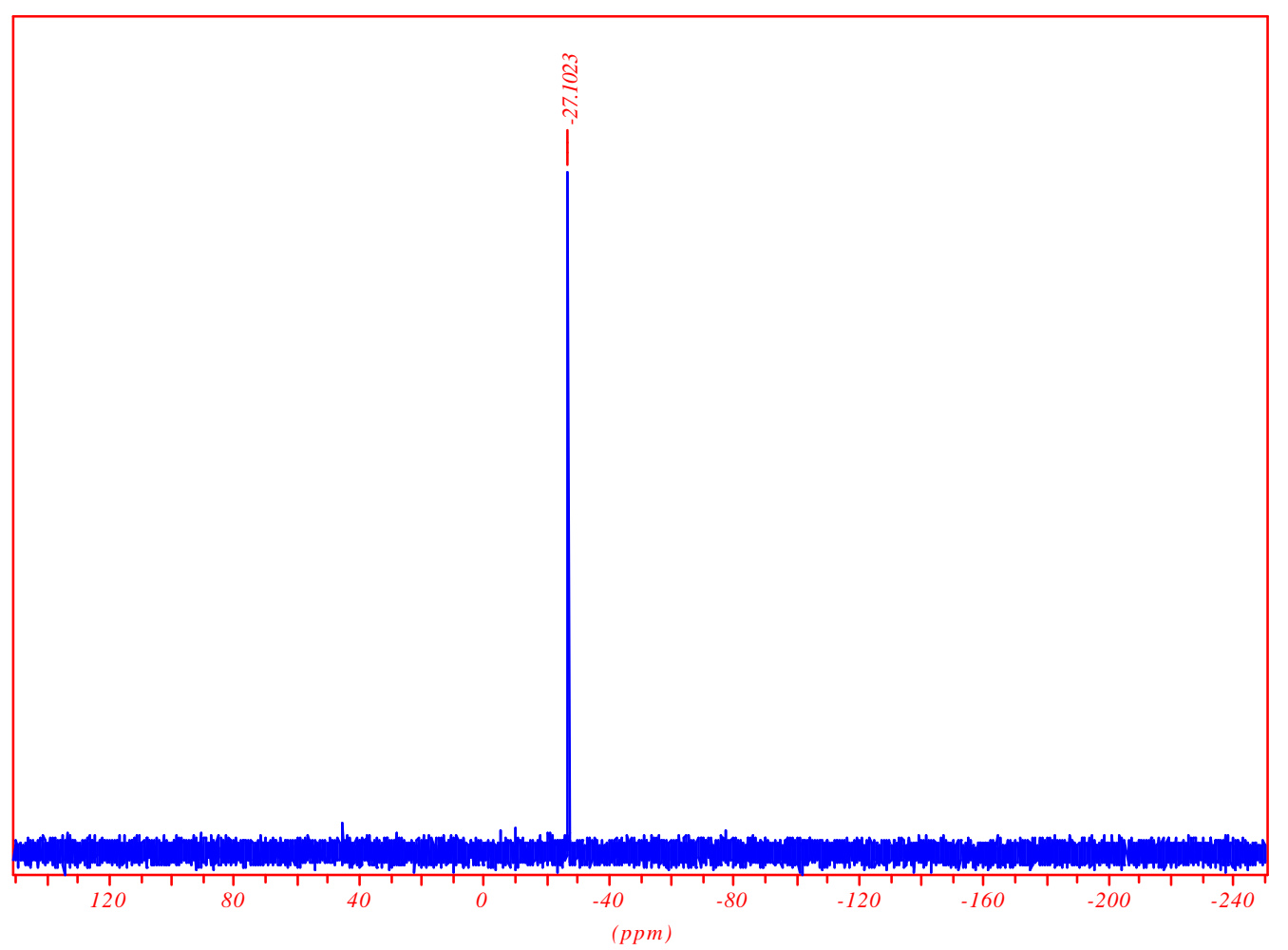


$\left[\mathbf{R}_{\mathrm{FP}}\left(\mathrm{CH}_{2}\right)_{3}\right]\left[\mathbf{R}_{\mathrm{F8}}\left(\mathrm{CH}_{2}\right)_{4}\right]_{2}\left(\mathrm{NC}-\mathrm{CH}_{2} \mathrm{CH}_{2}\right) \mathbf{P}^{+} \mathbf{I}^{-}$

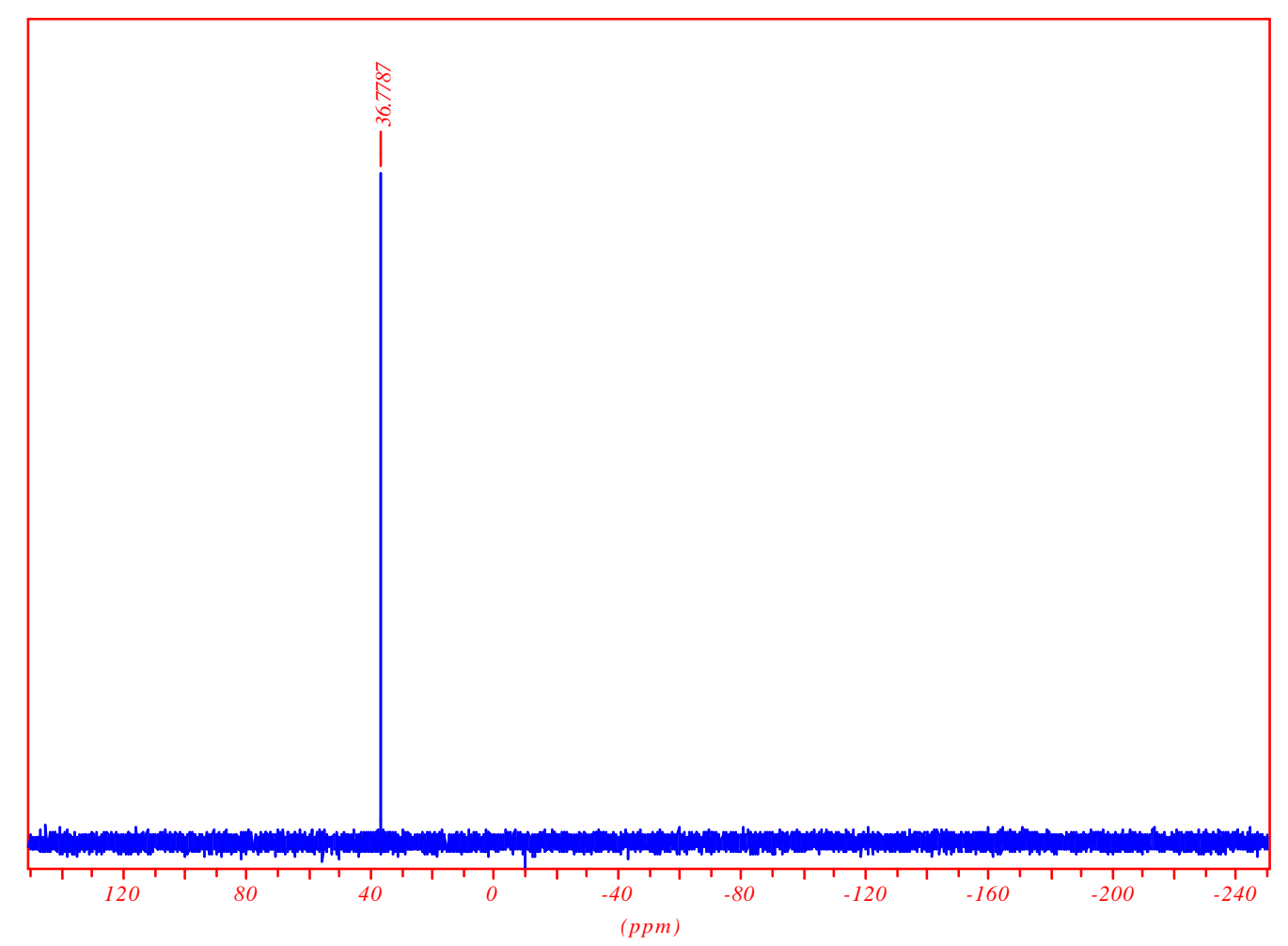


$\left[\mathbf{R}_{\mathrm{F8}}\left(\mathrm{CH}_{2}\right)_{3}\right]\left[\mathbf{R}_{\mathrm{F8}}\left(\mathrm{CH}_{2}\right)_{4}\right]_{2} \mathbf{P}$

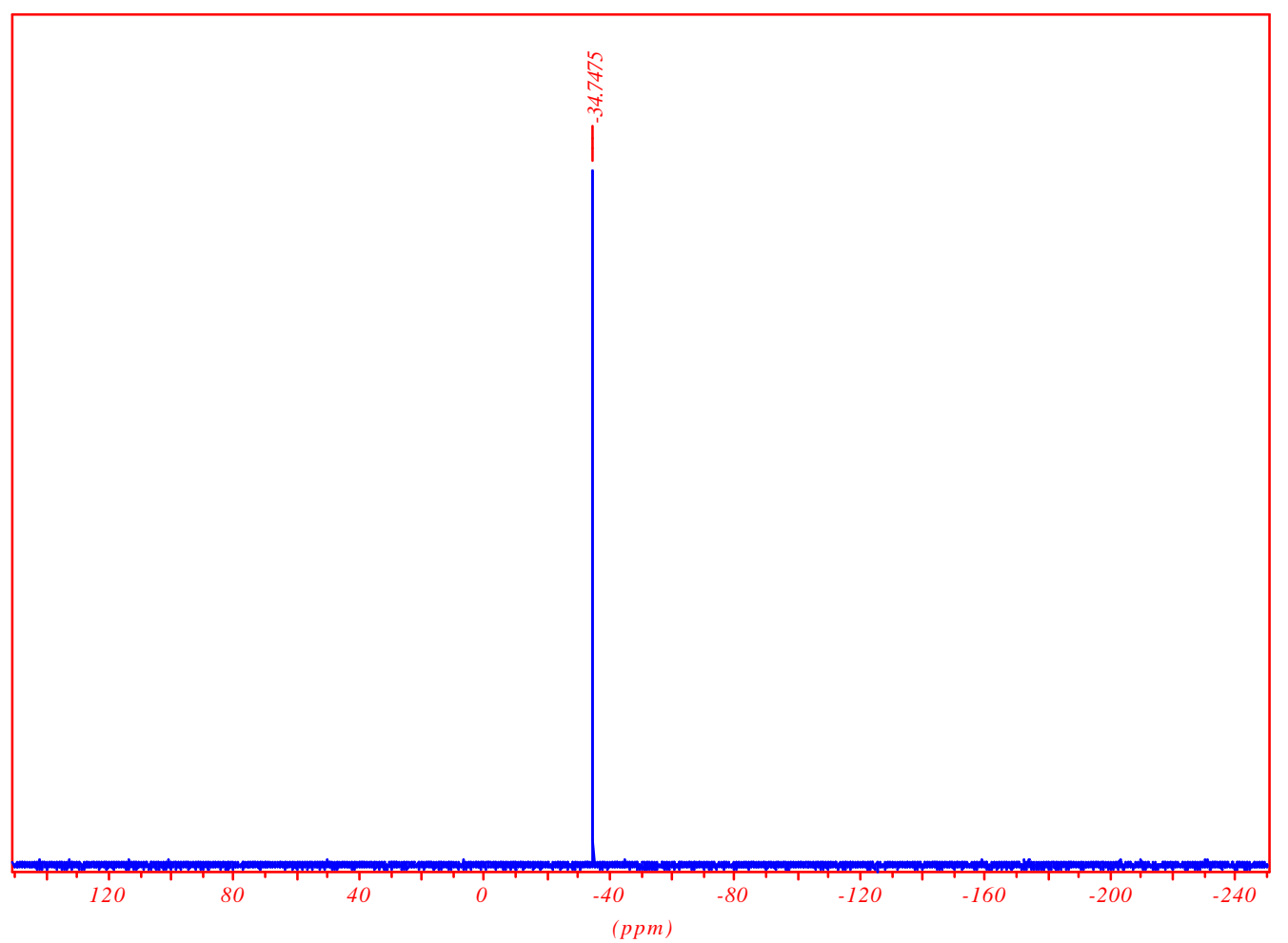


$\left[\mathrm{R}_{\mathrm{F6}}\left(\mathrm{CH}_{2}\right)_{3}\right]\left(\mathrm{NC}-\mathrm{CH}_{2} \mathrm{CH}_{2}\right)_{3} \mathbf{P}^{+} \mathbf{I}^{-}$

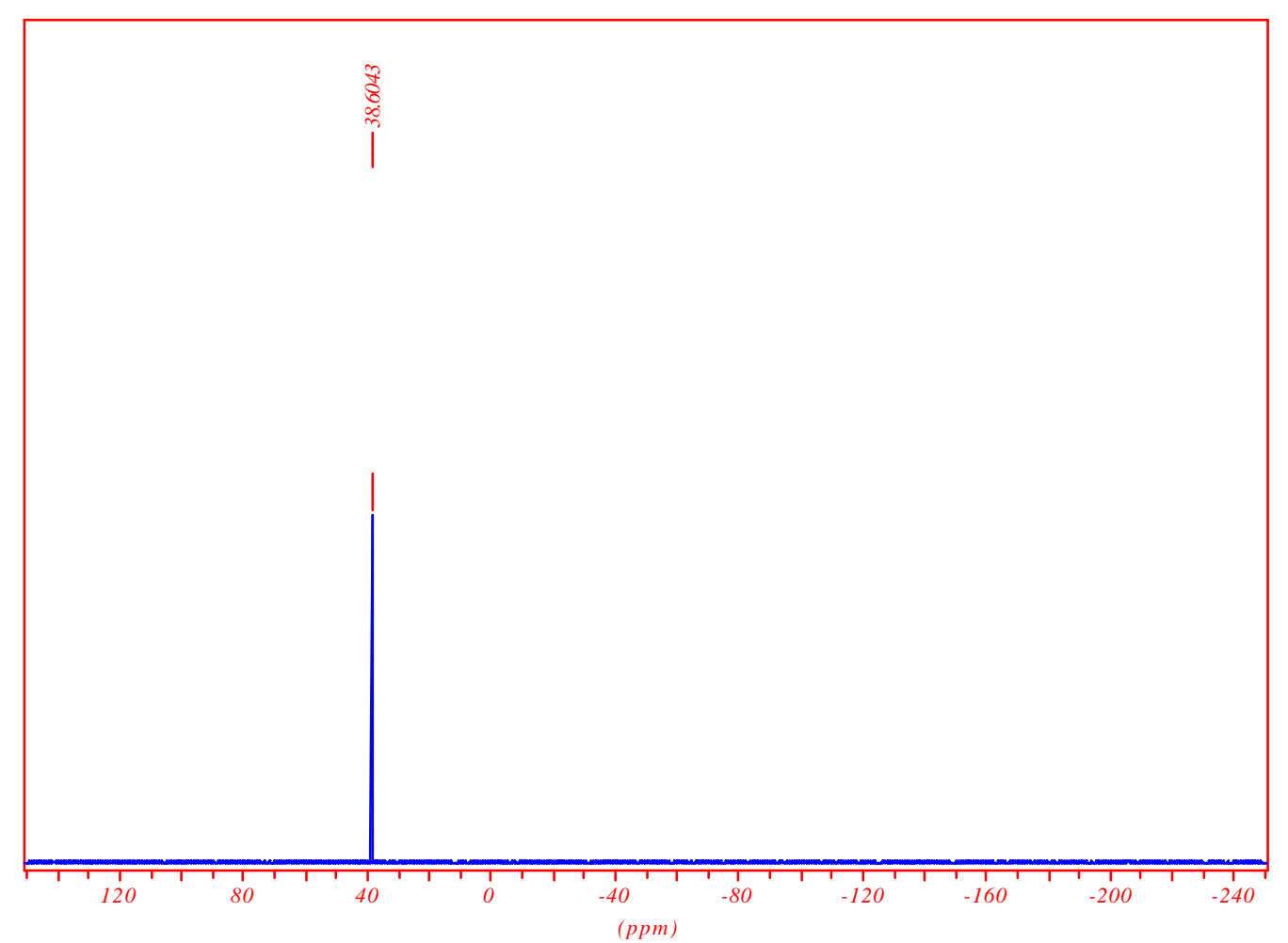


$\left[\mathbf{R}_{\mathrm{F} 6}\left(\mathrm{CH}_{2}\right)_{3}\right]\left(\mathrm{NC}-\mathrm{CH}_{2} \mathrm{CH}_{2}\right)_{2} \mathbf{P}$

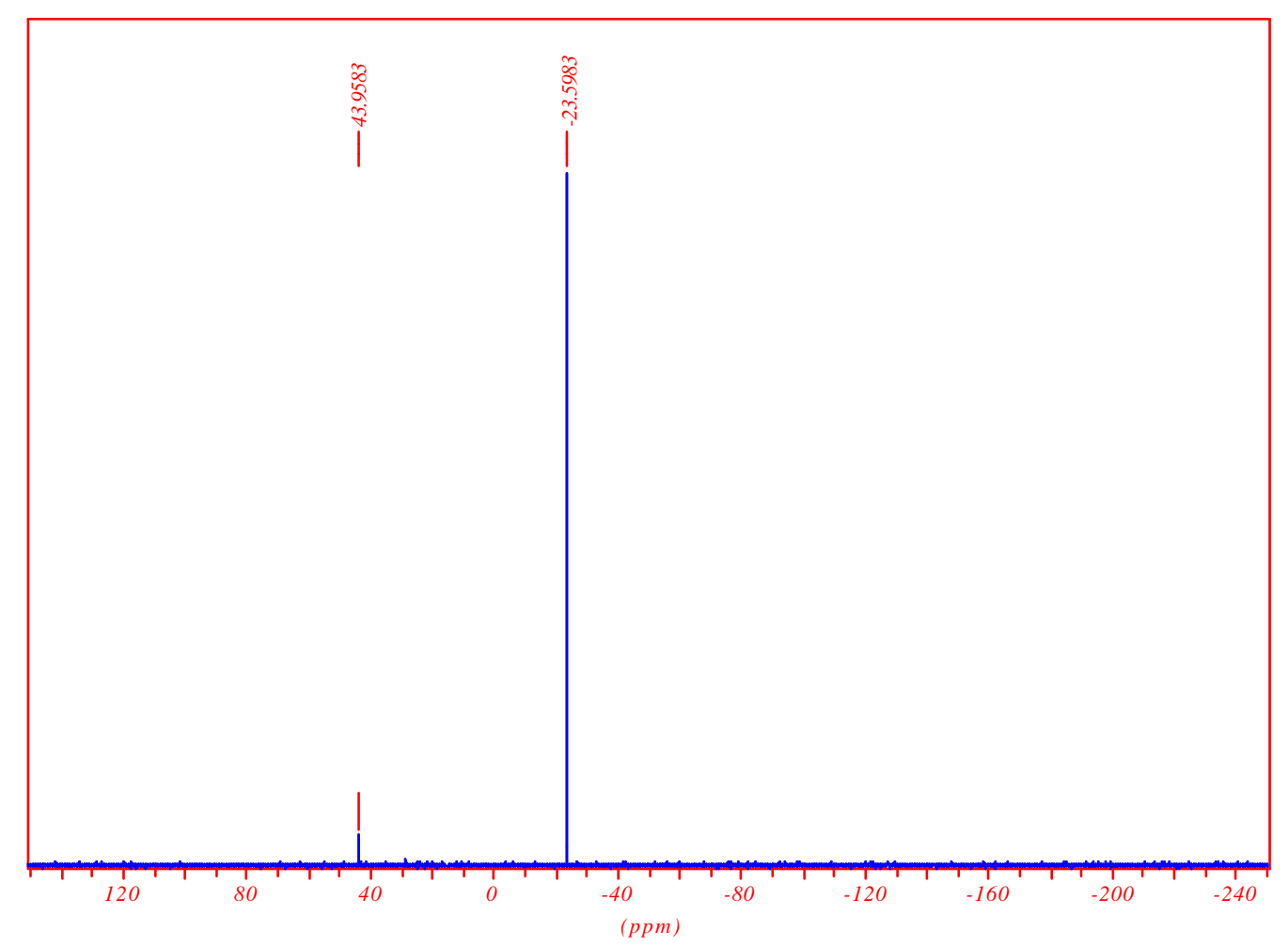


$\left[\mathbf{R}_{\mathrm{F6}}\left(\mathrm{CH}_{2}\right)_{3}\right]\left[\mathbf{R}_{\mathrm{F8}}\left(\mathrm{CH}_{2}\right)_{3}\right]\left(\mathrm{NC}-\mathrm{CH}_{2} \mathrm{CH}_{2}\right)_{2} \mathbf{P}^{+} \mathbf{I}^{-}$

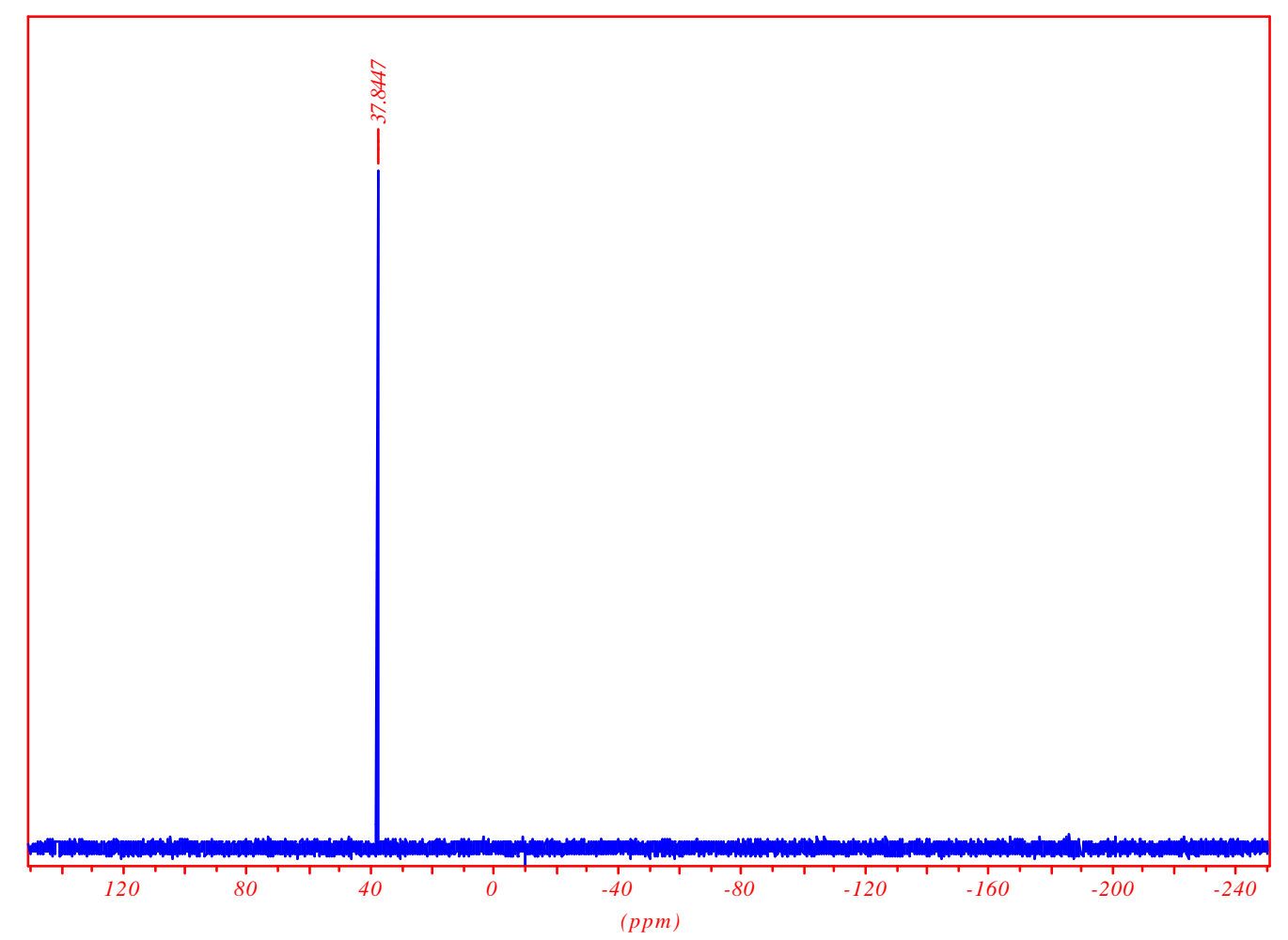


$\left[\mathbf{R}_{\mathrm{FG}}\left(\mathrm{CH}_{2}\right)_{3}\right]\left[\mathbf{R}_{\mathrm{F} 8}\left(\mathrm{CH}_{2}\right)_{3}\right]\left(\mathrm{NC}-\mathrm{CH}_{2} \mathrm{CH}_{2}\right) \mathbf{P}$

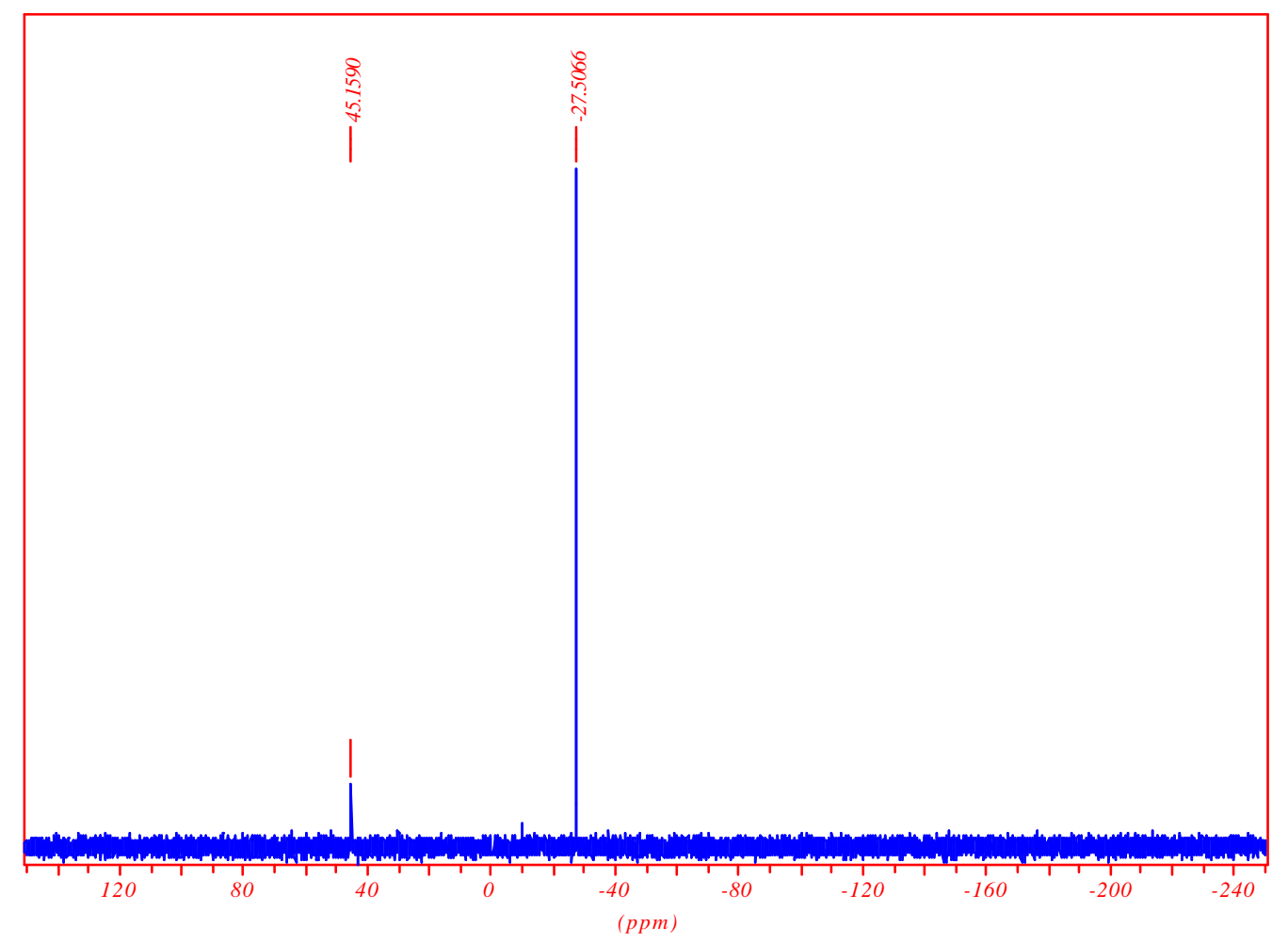


$\left[\mathbf{R}_{\mathrm{F6}}\left(\mathrm{CH}_{2}\right)_{3}\right]\left[\mathrm{R}_{\mathrm{F8}}\left(\mathrm{CH}_{2}\right)_{3}\right]\left[\mathrm{R}_{\mathrm{F8}}\left(\mathrm{CH}_{2}\right)_{4}\right]\left(\mathrm{NC}-\mathrm{CH}_{2} \mathrm{CH}_{2}\right) \mathbf{P}^{+} \mathbf{I}^{-}$

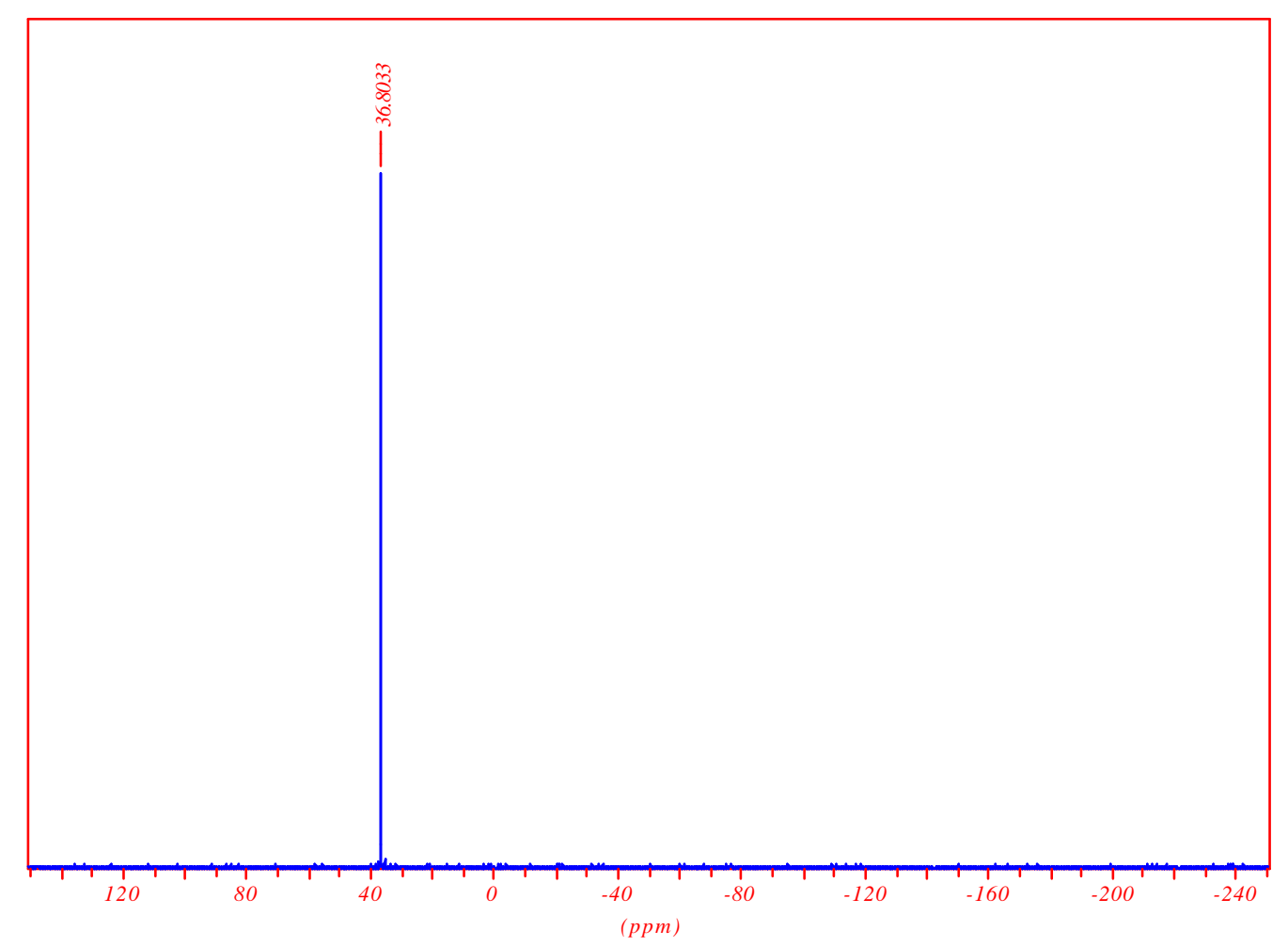


$\left[\mathbf{R}_{\mathrm{FF}}\left(\mathrm{CH}_{2}\right)_{3}\right]\left[\mathbf{R}_{\mathrm{F8}}\left(\mathrm{CH}_{2}\right)_{3}\right]\left[\mathbf{R}_{\mathrm{F8}}\left(\mathrm{CH}_{2}\right)_{4}\right] \mathbf{P}$

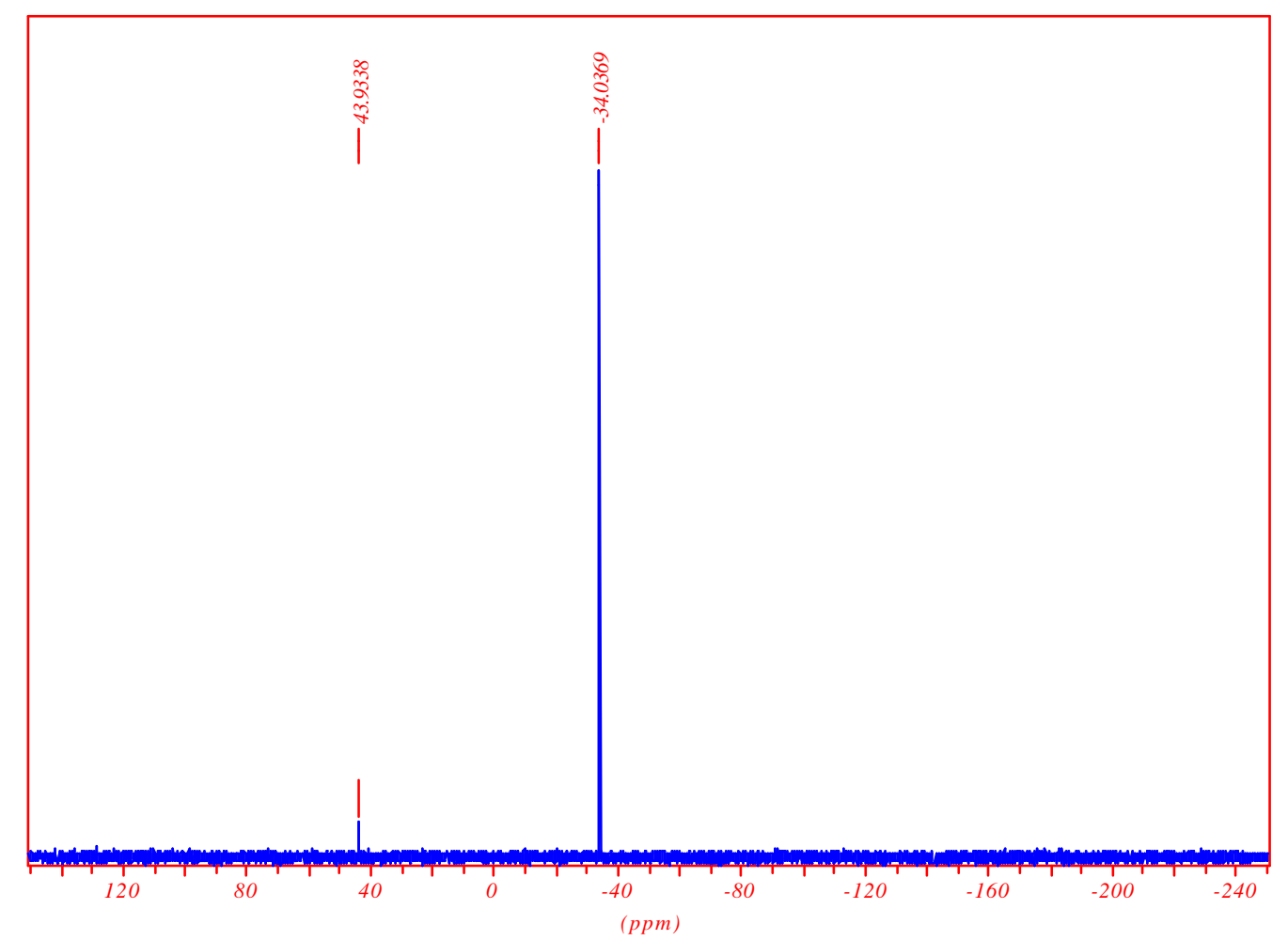

